CUBO A Mathematical Journal

Vol.20, $\mathrm{N}^{\beta \mathrm{o}}$ 2, (67-93). June 2018

\title{
Some remarks on the non-real roots of polynomials
}

\author{
Shuichi Otake ${ }^{1}$ and Tony Shaska ${ }^{2}$ \\ ${ }^{1}$ Department of Applied Mathematics, \\ Waseda University, \\ Japan. \\ ${ }^{2}$ Department of Mathematics and Statistics, \\ Oakland University, \\ Rochester, MI, 48309. \\ shuichi.otake.8655@gmail.com, shaska@oakland.edu
}

\begin{abstract}
Let $f \in \mathbb{R}(t)[x]$ be given by $f(t, x)=x^{n}+t \cdot g(x)$ and $\beta_{1}<\cdots<\beta_{m}$ the distinct real roots of the discriminant $\Delta_{(f, x)}(t)$ of $f(t, x)$ with respect to $x$. Let $\gamma$ be the number of real roots of $g(x)=\sum_{k=0}^{s} t_{s-k} x^{s-k}$. For any $\xi>\left|\beta_{m}\right|$, if $n-s$ is odd then the number of real roots of $f(\xi, x)$ is $\gamma+1$, and if $n-s$ is even then the number of real roots of $f(\xi, x)$ is $\gamma, \gamma+2$ if $t_{s}>0$ or $t_{s}<0$ respectively. A special case of the above result is constructing a family of degree $n \geq 3$ irreducible polynomials over $\mathbb{Q}$ with many non-real roots and automorphism group $S_{n}$.
\end{abstract}

\section{RESUMEN}

Sea $f \in \mathbb{R}(t)[x]$ dada por $f(t, x)=x^{n}+t \cdot g(x)$ y $\beta_{1}<\cdots<\beta_{m}$ las diferentes raíces reales del discriminante $\Delta_{(f, x)}(t)$ de $f(t, x)$ con respecto de $x$. Sea $\gamma$ el número de raíces reales de $g(x)=\sum_{k=0}^{s} t_{s-k} x^{s-k}$. Para todo $\xi>\left|\beta_{m}\right|$, si $n-s$ es impar entonces el número de raíces reales de $f(\xi, x)$ es $\gamma+1$, y si $n-s$ es par entonces el número de raíces reales de $f(\xi, x)$ es $\gamma, \gamma+2$ si $t_{s}>0$ o $t_{s}<0$, respectivamente. Un caso especial del resultado anterior es construyendo una familia de polinomios irreducibles sobre $\mathbb{Q}$ de grado $n \geq 3$ con muchas raíces no-reales y grupo de automorfismos $S_{n}$

Keywords and Phrases: Polynomials, non-real roots, discriminant, Bezoutian, Galois groups.

2010 AMS Mathematics Subject Classification: 12D10, 12F10, 26C10. 


\section{Introduction}

Let $f(x) \in \mathbb{Q}[x]$ be an irreducible polynomial of degree $n \geq 2$ and Gal (f) its Galois group over $\mathbb{Q}$. Let us assume that over $\mathbb{R}, f(x)$ is factored as

$$
f(x)=a \prod_{j=1}^{r}\left(x-\alpha_{j}\right) \prod_{i=1}^{s}\left(x^{2}+a_{i} x+b_{i}\right),
$$

where $a_{i}^{2}<4 b_{i}$, for all $i=1, \ldots, s$. The pair $(r, s)$ is called the signature of $f(x)$. Obviously $\operatorname{deg} f=2 s+r$. If $s=0$ then $f(x)$ is called totally real and if $r=0$ it is called totally complex. Equivalently the above terminology can be defined for binary forms $f(x, z)$. By a reordering of the roots we may assume that if $f(x)$ has $2 s$ non-real roots then

$$
\alpha:=(1,2)(3,4) \cdots(2 s-1,2 s) \in \operatorname{Gal}(f) .
$$

In 4 it is proved that if $\operatorname{deg} f=p$, for a prime $p$, and $s$ satisfies

$$
s(s \log s+2 \log s+3) \leq p
$$

then $\operatorname{Gal}(f)=A_{p}, S_{p}$. Moreover, a list of all possible groups for various values of $r$ is given for $p \leq 29$; see [4, Thm. 2]. There are some follow up papers to [4].

In [1] the author proves that if $p \geq 4 s+1$, then the Galois group is either $S_{p}$ or $A_{p}$. This improves the bound given in [4]. The author also studies when polynomials with non-real roots are solvable by radicals, which are consequences of Table 2 and Theorem 2 in [4]. In [13] the author uses Bezoutians of a polynomial and its derivative to construct polynomials with real coefficients where the number of real roots can be counted explicitly. Thereby, irreducible polynomials in $\mathbb{Q}[x]$ of prime degree $p$ are constructed for which the Galois group is either $S_{p}$ or $A_{p}$.

In this paper we study a family of polynomials with non-real roots whose degree is not necessarily prime. Given a polynomial $g(x)=\sum_{i=0}^{s} t_{i} x^{i}$ and with $\gamma$ number of non-real roots we construct a polynomial $\mathrm{f}(\mathrm{t}, \mathrm{x})=\mathrm{x}^{\mathrm{n}}+\mathrm{tg}(\mathrm{x})$ which has $\gamma, \gamma+1, \gamma+2$ non-real roots for certain values of $t \in \mathbb{R}$; see Theorem 3.2. The values of $t \in \mathbb{R}$ are given in terms of the Bezoutian matrix of polynomials or equivalently the discriminant of $f(t, x)$ with respect to $x$. This is the focus of Section 3 in the paper.

While most of the efforts have been focusing on the case of irreducible polynomials over $\mathbb{Q}$ which have real roots, the case of polynomials with no real roots is equally interesting. How should an irreducible polynomial over $\mathbb{Q}$ with all non-real roots must look like? What can be said about the Galois group of such totally complex polynomials? In [5] is developed a reduction theory for such polynomials via the hyperbolic center of mass. A special case of Theorem 3.2 provides a class of totally complex polynomials.

Notation For any polynomial $f(x)$ we denote by $\Delta_{(f, x)}$ its discriminant with respect to $x$. If $f$ is a univariate polynomial then $\Delta_{\mathrm{f}}$ is used and the leading coefficient is denoted by led(f). Throughout this paper the ground field is a field of characteristic zero. 


\section{Preliminaries}

Let $f_{1}(x), f_{2}(x)$ be polynomials over a field $F$ of characteristic zero and, let $n$ be an integer which is greater than or equal to $\max \left\{\operatorname{deg} f_{1}, \operatorname{deg} f_{2}\right\}$. Then, we put

$$
\begin{aligned}
& B_{n}\left(f_{1}, f_{2}\right):=\frac{f_{1}(x) f_{2}(y)-f_{1}(y) f_{2}(x)}{x-y}=\sum_{i, j=1}^{n} \alpha_{i j} x^{n-i} y^{n-j} \in F[x, y], \\
& M_{n}\left(f_{1}, f_{2}\right):=\left(\alpha_{i j}\right)_{1 \leq i, j \leq n} .
\end{aligned}
$$

The matrix $M_{n}\left(f_{1}, f_{2}\right)$ is called the Bezoutian of $f_{1}$ and $f_{2}$. Clearly, $B_{n}\left(f_{1}, f_{1}\right)=0$ and hence $M_{n}\left(f_{1}, f_{1}\right)$ is the zero matrix. The following properties hold true; see [6, Theorem 8.25] for details.

Proposition 1. The following are true:

(1) $M_{n}\left(f_{1}, f_{2}\right)$ is an $n \times n$ symmetric matrix over $F$.

(2) $B_{n}\left(f_{1}, f_{2}\right)$ is linear in $f_{1}$ and $f_{2}$, separately.

(3) $B_{n}\left(f_{1}, f_{2}\right)=-B_{n}\left(f_{2}, f_{1}\right)$.

When $f_{2}=f_{1}^{\prime}$, the formal derivative of $f_{1}$ (with respect to the indeterminate $x$ ), we often write $B_{n}\left(f_{1}\right):=B_{n}\left(f_{1}, f_{1}^{\prime}\right)$. From now on, for any degree $n \geq 2$ polynomial $f(x) \in \mathbb{R}[x]$ we will denote by $M_{n}(f):=M_{n}\left(f, f^{\prime}\right)$ as above. The matrix $M_{n}(f)$ is called the Bezoutian matrix of $f$.

Remark 2.1. It is often the case that the matrix $M_{n}^{\prime}\left(f_{1}, f_{2}\right)=\left(\alpha_{i j}^{\prime}\right)_{1 \leq i, j \leq n}$ defined by the generating function

$$
B_{n}^{\prime}\left(f_{1}, f_{2}\right):=\frac{f_{1}(x) f_{2}(y)-f_{1}(y) f_{2}(x)}{x-y}=\sum_{i, j=1}^{n} \alpha_{i j}^{\prime} x^{i-1} y^{j-1} \in F[x, y]
$$

is called the Bezoutian of $\mathrm{f}_{1}$ and $\mathrm{f}_{2}$. But no difference can be seen between these two definitions as far as we consider the corresponding quadratic forms

$$
\sum_{i, j=1}^{n} \alpha_{i j} x_{i} x_{j} \text { and } \sum_{i, j=1}^{n} \alpha_{i j}^{\prime} x_{i} x_{j} .
$$

In fact, these two quadratic forms are equivalent over the prime field $\mathbb{Q}(\subset \mathrm{F})$ since we have $M_{n}^{\prime}\left(f_{1}, f_{2}\right)={ }^{t} J_{n} M_{n}\left(f_{1}, f_{2}\right) J_{n}$, where

$$
J_{n}=\left[\begin{array}{cccc}
0 & & & 1 \\
& . \cdot & & \\
1 & & & 0
\end{array}\right]
$$

is an $\mathrm{n} \times \mathrm{n}$ anti-identity matrix. This implies that above two quadratic forms are equivalent over $\mathbb{Q}$ or more precisely, over the ring of rational integers $\mathbb{Z}$. 
Let $f(x) \in \mathbb{R}[x]$ be a degree $n \geq 2$ polynomial which is given by

$$
f(x)=a_{0}+a_{1} x+\cdots+a_{n} x^{n}
$$

Then over $\mathbb{R}$ this polynomial is factored as

$$
f(x)=a \prod_{j=1}^{r}\left(x-\alpha_{j}\right) \prod_{i=1}^{s}\left(x^{2}+a_{i} x+b_{i}\right)
$$

for some $\alpha_{1}, \ldots, \alpha_{r} \in \mathbb{R}$ and $a_{i}, b_{i}, a \in \mathbb{R}$, where $a_{i}^{2}<4 b_{i}$, for all $i=1, \cdots, s$.

Throughout this paper, for a univariate polynomial $\mathrm{f}$, its discriminant will be denoted by $\Delta_{\mathrm{f}}$. For any two polynomials $f_{1}(x), f_{2}(x)$ the resultant with respect to $x$ will be denoted by $\operatorname{Res}\left(f_{1}, f_{2}, x\right)$. We notice the following elementary fact, its proof is elementary and we skip the details.

Remark 2.2. For any polynomial $\mathrm{f}(\mathrm{x})$, the determinant of the Bezoutian is the same as the discriminant up to a multiplication by a constant. More precisely,

$$
\Delta_{\mathrm{f}}=\frac{1}{l e d(\mathrm{f})^{2}} \operatorname{det} M_{\mathrm{n}}(\mathrm{f}),
$$

where led(f) is the leading coefficient of $\mathrm{f}(\mathrm{x})$.

If $f(x) \in \mathbb{Q}[x]$ is irreducible and its degree is a prime number, say $\operatorname{deg} f=p$, then there is enough known for the Galois group of polynomials with some non-real roots; see [4, [1], [13] for details. If the number of non-real roots is "small" enough with respect to the prime $\operatorname{degree} \operatorname{deg} f=p$ of the polynomial, then the Galois group is $A_{p}$ or $S_{p}$. Furthermore, using the classification of finite simple groups one can provide a complete list of possible Galois groups for every polynomial of prime degree $p$ which has non-real roots; see [4] for details.

On the other extreme are the polynomials which have all roots non-real. We called them above, totally complex polynomials. We have the following:

Lemma 2.1. The followings are equivalent:

i) $\mathrm{f}(\mathrm{x}) \in \mathbb{R}[\mathrm{x}]$ is totally complex

ii) $\mathrm{f}(\mathrm{x})$ can be written as

$$
f(x)=a \prod_{i=1}^{n} f_{i}
$$

where $f_{i}=x^{2}+a_{i} x+b_{i}$, for $i=1, \ldots, n$ and $a_{i}, b_{i}, a \in \mathbb{R}$, where $a_{i}^{2}<4 b_{i}$, for all $i=1, \ldots, n$. Moreover, the determinant of the Bezoutian $\mathrm{M}_{\mathrm{n}}(\mathrm{f})$ is given by

$$
\Delta_{\mathrm{f}}=\frac{1}{\operatorname{led}(\mathrm{f})^{2}} \operatorname{det} M_{\mathrm{n}}(\mathrm{f})=\prod_{i=1}^{\mathrm{n}} \Delta_{\mathrm{f}_{i}} \cdot \prod_{i, j, i \neq j}^{n}\left(\operatorname{Res}\left(\mathrm{f}_{\mathrm{i}}, \mathrm{f}_{\mathrm{j}}, \mathrm{x}\right)\right)^{2}
$$


where led(f) is the leading coefficient of $\mathrm{f}(\mathrm{x})$.

ii) the index of inertia of Bezoutian $\mathrm{M}(\mathrm{f})$ is 0

iii) if $\Delta_{\mathrm{f}} \neq 0$ then the equivalence class of $\mathrm{M}(\mathrm{f})$ in the Witt ring $\mathrm{W}(\mathrm{R})$ is 0 .

Proof. The equivalence between i), ii), and iii) can be found in [6].

It is not clear when such polynomials are irreducible over $\mathbb{Q}$. If that's the case, what is the Galois group Gal (f)? Clearly the group generated by the involution $(1,2)(3,4) \cdots(2 n-1,2 n)$ is embedded in Gal (f). Is Gal (f) larger in general?

\section{On the number of real roots of polynomials}

For any degree $n \geq 2$ polynomial $f(x) \in \mathbb{R}[x]$ and any symmetric matrix $M:=M_{n}(f)$ with real entries, let $N_{f}$ be the number of distinct real roots of $f$ and $\sigma(M)$ be the index of inertia of $M$, respectively. The next result plays a fundamental role throughout this section ([6, Theorem 9.2]).

Proposition 2. For any real polynomial $\mathrm{f} \in \mathbb{R}[\mathrm{x}]$, the number $\mathrm{N}_{\mathrm{f}}$ of its distinct real roots is the index of inertia of the Bezoutian matrix $\mathrm{M}_{\mathrm{n}}(\mathrm{f})$. In other words,

$$
N_{f}=\sigma\left(M_{n}(f)\right) .
$$

Let us cite one more result which says that the roots of a polynomial depend continuously on its coefficients (11, Theorem 1.4], [16, Theorem 1.3.1]).

Proposition 3. Let be given a polynomial

$$
f(x)=\sum_{l=0}^{n} a_{l} x^{l} \in \mathbb{C}[x]
$$

with distinct roots $\alpha_{1}, \ldots, \alpha_{k}$ of multiplicities $\mathrm{m}_{1}, \ldots, \mathrm{m}_{\mathrm{k}}$ respectively. Then, for any given a positive

$$
\varepsilon<\min _{1 \leq i<j \leq k}\left\{\frac{\left|\alpha_{i}-\alpha_{j}\right|}{2}\right\}
$$

there exists a real number $\delta>0$ such that any monic polynomial $\mathrm{g}(\mathrm{x})=\sum_{l=0}^{n} \mathrm{~b}_{l} \mathrm{x}^{\mathrm{l}} \in \mathbb{C}[\mathrm{x}]$ whose coefficients satisfy

$$
\left|b_{l}-a_{l}\right|<\delta,
$$

for $\mathrm{l}=0, \cdots, \mathrm{n}-1$, has exactly $\mathrm{m}_{\mathfrak{j}}$ roots in the disk

$$
\mathcal{D}\left(\alpha_{j} ; \varepsilon\right)=\left\{z \in \mathbb{C}|| z-\alpha_{j} \mid<\epsilon\right\}(j=1, \cdots, k) .
$$


Let $n, s$ be positive integers such that $n>s$ and let

$$
\begin{aligned}
g\left(t_{0}, \cdots, t_{s} ; x\right) & =\sum_{k=0}^{s} t_{s-k} x^{s-k}, \\
f^{(n)}\left(t_{0}, \cdots, t_{s}, t ; x\right) & =x^{n}+t \cdot g\left(t_{0}, \cdots, t_{s} ; x\right)
\end{aligned}
$$

be polynomials in $x$ over $E_{1}=\mathbb{R}\left(t_{0}, \cdots, t_{s}\right), E_{2}=\mathbb{R}\left(t_{0}, \cdots, t_{s}, t\right)$, respectively. Here, $E_{1}$ (resp., $E_{2}$ ) is a rational function field with $s+1$ (resp., $(s+2)$ ) variables $t_{0}, \cdots, t_{s}\left(\right.$ resp., $\left.\left(t_{0}, \cdots, t_{s}, t\right)\right)$. To ease notation, let us put

$$
g(x)=g\left(t_{0}, \cdots, t_{s} ; x\right), f(t ; x)=f^{(n)}\left(t_{0}, \cdots, t_{s}, t ; x\right)
$$

and for any real vector $v=\left(v_{0}, \cdots, v_{s}\right) \in \mathbb{R}^{s+1}$, we put

$$
g_{v}(x)=g\left(v_{0}, \cdots, v_{s} ; x\right), f_{v}(t ; x)=f^{(n)}\left(v_{0}, \cdots, v_{s}, t ; x\right) \text {. }
$$

By using Proposition 2, we can prove the next theorem ([13, Main Theorem 1.3]).

Theorem 3.1. Let $\mathrm{r}=\left(\mathrm{r}_{0}, \cdots, \mathrm{r}_{\mathrm{s}}\right) \in \mathbb{R}^{\mathrm{s}+1}$ be a vector such that $\mathrm{N}_{\mathrm{g}_{\mathrm{r}}}=\mathrm{s}$. Let us consider $\mathrm{f}_{\mathrm{r}}(\mathrm{t} ; \mathrm{x})=\mathrm{f}^{(\mathrm{n})}\left(\mathrm{r}_{\mathrm{O}}, \cdots, \mathrm{r}_{\mathrm{s}}, \mathrm{t} ; \mathrm{x}\right)$ as a polynomial over $\mathbb{R}(\mathrm{t})$ in $\mathrm{x}$ and put

$$
P_{r}(t)=\operatorname{det} M_{n}\left(f_{r}(t ; x)\right)=\operatorname{det} M_{n}\left(f_{r}(t ; x), f_{r}^{\prime}(t ; x)\right)
$$

where $\mathrm{f}_{\mathrm{r}}^{\prime}(\mathrm{t} ; \mathrm{x})$ is a derivative of $\mathrm{f}_{\mathrm{r}}(\mathrm{t} ; \mathrm{x})$ with respect to $\mathrm{x}$. Then, for any real number $\xi>\alpha_{\mathrm{r}}=$ $\max \left\{\alpha \in \mathbb{R} \mid \mathrm{P}_{\mathrm{r}}(\alpha)=0\right\}$, we have

$$
\mathrm{N}_{\mathrm{f}_{\mathrm{r}}(\xi ; \mathrm{x})}= \begin{cases}\mathrm{s}+1 & \text { if } \mathrm{n}-\mathrm{s}: \text { odd } \\ \mathrm{s} & \text { if } \mathrm{n}-\mathrm{s}: \text { even, } \mathrm{r}_{\mathrm{s}}>0 \\ \mathrm{~s}+2 & \text { if } \mathrm{n}-\mathrm{s}: \text { even, } \mathrm{r}_{\mathrm{s}}<0\end{cases}
$$

By this theorem and a theorem of Oz Ben-Shimol [1, Theorem 2.6], we can obtain an algorithm to construct prime degree $p$ polynomials with given number of real roots, and whose Galois groups are isomorphic to the symmetric group $S_{p}$ or the alternating group $A_{p}$ ([13, Corollary 1.6]).

In this section, we extend this theorem as follows;

Theorem 3.2. Let $\mathrm{r}=\left(\mathrm{r}_{0}, \cdots, \mathrm{r}_{\mathrm{s}}\right) \in \mathbb{R}^{\mathrm{s}+1}$ be a vector such that $\mathrm{g}_{\mathrm{r}}(\mathrm{x})$ is a degree separable polynomial satisfying $\mathrm{N}_{\mathrm{g}_{\mathrm{r}}(\mathrm{x})}=\gamma(0 \leq \gamma \leq \mathrm{s})$. Let us consider $\mathrm{f}_{\mathrm{r}}(\mathrm{t} ; \mathrm{x})=\mathrm{f}^{(\mathrm{n})}\left(\mathrm{r}_{\mathrm{O}}, \cdots, \mathrm{r}_{\mathrm{s}}, \mathrm{t} ; \mathrm{x}\right)$ as a polynomial over $\mathbb{R}(\mathrm{t})$ in $\mathrm{x}$ and put

$$
P_{r}(t)=\operatorname{det} M_{n}\left(f_{r}(t ; x)\right)=\operatorname{det} M_{n}\left(f_{r}(t ; x), f_{r}^{\prime}(t ; x)\right)
$$

where $\mathrm{f}_{\mathrm{r}}^{\prime}(\mathrm{t} ; \mathrm{x})$ is a derivative of $\mathrm{f}_{\mathrm{r}}(\mathrm{t} ; \mathrm{x})$ with respect to $\mathrm{x}$. Then, for any real number $\xi>\alpha_{\mathrm{r}}=$ $\max \left\{\alpha \in \mathbb{R} \mid \mathrm{P}_{\mathrm{r}}(\alpha)=0\right\}$, we have

$$
\mathrm{N}_{\mathrm{f}_{\mathrm{r}}(\xi ; \mathrm{x})}= \begin{cases}\gamma+1 & \text { if } \mathrm{n}-\mathrm{s}: \text { odd } \\ \gamma & \text { if } \mathrm{n}-\mathrm{s}: \text { even, } \mathrm{r}_{\mathrm{s}}>0 \\ \gamma+2 & \text { if } \mathrm{n}-\mathrm{s}: \text { even, } \mathrm{r}_{\mathrm{s}}<0\end{cases}
$$


The above theorem can be restated as follows:

Corolary 1. Let $\mathbf{f} \in \mathbb{R}(\mathrm{t})[\mathrm{x}]$ be given by

$$
f(t, x)=x^{n}+t \cdot \sum_{k=0}^{s} t_{s-k} x^{s-k}
$$

and $\beta_{1}<\cdots<\beta_{\mathrm{m}}$ the distinct real roots of the degree $\mathrm{s}$ polynomial

$$
\mathrm{P}(\mathrm{t}):=\frac{1}{\mathrm{t}^{\mathrm{n}-1}} \Delta_{(\mathrm{f}, \mathrm{x})}(\mathrm{t}) .
$$

For any $\xi>\left|\beta_{m}\right|$, the number of real roots of $\mathrm{f}(\xi, x)$ is

$$
\mathrm{N}_{\mathrm{f}(\xi, x)}= \begin{cases}\gamma+1 & \text { if } \mathrm{n}-\mathrm{s}: \text { odd } \\ \gamma & \text { if } \mathrm{n}-\mathrm{s}: \text { even, } \mathrm{t}_{\mathrm{s}}>0 \\ \gamma+2 & \text { if } \mathrm{n}-\mathrm{s}: \text { even, } \mathrm{t}_{\mathrm{s}}<0 .\end{cases}
$$

where $\gamma$ is the number or real roots of $\mathrm{g}(\mathrm{x})=\frac{\mathrm{f}(\mathrm{x})-\mathrm{x}^{\mathrm{n}}}{\mathrm{t}} \in \mathbb{R}[\mathrm{x}]$.

The rest of the section is concerned with proving Thm. 3.2 .

\subsection{The Bezoutian of $f(t ; x)$}

First, let us put

$$
\begin{aligned}
& A\left(t_{0}, \cdots, t_{s}, t\right)=\left(a_{i j}\left(t_{0}, \cdots, t_{s}, t\right)\right)_{1 \leq i, j \leq n}=M_{n}(f(t ; x)) \in \operatorname{Sym}_{n}\left(E_{2}\right), \\
& B\left(t_{0}, \cdots, t_{s}\right)=\left(b_{i j}\left(t_{0}, \cdots, t_{s}\right)\right)_{1 \leq i, j \leq s}=M_{s}(g(x)) \in \operatorname{Sym}_{s}\left(E_{1}\right) .
\end{aligned}
$$

For ease of notation, we also write

$$
A\left(t_{0}, \cdots, t_{s}, t\right)=A(t)=\left(a_{i j}(t)\right)_{1 \leq i, j \leq n}, B\left(t_{0}, \cdots, t_{s}\right)=B=\left(b_{i j}\right)_{1 \leq i, j \leq s}
$$

and we put $B(t)=\left(b_{i j}(t)\right)_{1 \leq i, j \leq s}=t^{2} B$. Then, by Proposition 1 we have

$$
\begin{aligned}
A(t)= & M_{n}\left(x^{n}+t g(x), n x^{n-1}+t g^{\prime}(x)\right) \\
= & n M_{n}\left(x^{n}, x^{n-1}\right)-n t M_{n}\left(x^{n-1}, g(x)\right)+t M_{n}\left(x^{n}, g^{\prime}(x)\right)+t^{2} M_{n}\left(g(x), g^{\prime}(x)\right) \\
= & n M_{n}\left(x^{n}, x^{n-1}\right)-n t \sum_{k=0}^{s} t_{s-k} M_{n}\left(x^{n-1}, x^{s-k}\right) \\
& \quad+t \sum_{k=0}^{s-1}(s-k) t_{s-k} M_{n}\left(x^{n}, x^{s-k-1}\right)+t^{2} M_{n}\left(g(x), g^{\prime}(x)\right) .
\end{aligned}
$$

Lemma 3.1. Let $\lambda, \mu, v$ be integers such that $\lambda \geq \mu>v \geq 0$. Then $M_{\lambda}\left(\chi^{\mu}, x^{v}\right)=\left(m_{i j}\right)_{1 \leq i, j \leq \lambda}$, where

$$
m_{i j}= \begin{cases}1 & i+j=2 \lambda-(\mu+v)+1 \quad(\lambda-\mu+1 \leq i, j \leq \lambda-v), \\ 0 & \text { otherwise. }\end{cases}
$$


Proof. By definition, we have

$$
\begin{aligned}
B_{\lambda}\left(x^{\mu}, x^{\nu}\right) & =\frac{x^{\mu} y^{\nu}-x^{\nu} y^{\mu}}{x-y} \\
& =\sum_{k=1}^{\mu-v} x^{\mu-k} y^{\nu+k-1}=\sum_{k=1}^{\mu-v} x^{\lambda-(\lambda-\mu+k)} y^{\lambda-(\lambda-v-k+1)},
\end{aligned}
$$

which implies

$$
\begin{aligned}
m_{i j} & = \begin{cases}1 & (i, j)=(\lambda-\mu+k, \lambda-v-k+1) \quad(1 \leq k \leq \mu-v) \\
0 & \text { otherwise }\end{cases} \\
& = \begin{cases}1 & i+j=2 \lambda-(\mu+v)+1 \quad(\lambda-\mu+1 \leq i, j \leq \lambda-v), \\
0 & \text { otherwise }\end{cases}
\end{aligned}
$$

This completes the proof.

Here, let us divide $A(t)$ into two parts $\hat{A}(t)$ and $\tilde{A}(t)$, where

$$
\begin{aligned}
& \hat{A}(t)=\left(\widehat{a}_{i j}(t)\right)_{1 \leq i, j \leq n}=n M_{n}\left(x^{n}, x^{n-1}\right)-n t \sum_{k=0}^{s} t_{s-k} M_{n}\left(x^{n-1}, x^{s-k}\right) \\
&+t \sum_{k=0}^{s-1}(s-k) t_{s-k} M_{n}\left(x^{n}, x^{s-k-1}\right), \\
& \tilde{A}(t)=\left(\tilde{a}_{i j}(t)\right)_{1 \leq i, j \leq n}=t^{2} M_{n}\left(g(x), g^{\prime}(x)\right)
\end{aligned}
$$

and put $l_{k}=n-s+k+2(=2 n-(n+s-k-1)+1)$. Then, by lemma 3.1, we have

$$
\left\{\begin{array}{l}
\hat{a}_{11}(t)=n \\
\hat{a}_{1, l_{k}-1}(t)=\hat{a}_{l_{k}-1,1}(t)=(s-k) t_{s-k} t(0 \leq k \leq s-1) .
\end{array}\right.
$$

Moreover, when $i+j=l_{k}$, we have

$$
\hat{\mathrm{a}}_{i j}(\mathrm{t})=-n \mathrm{nt} \mathrm{t}_{s-k}+\mathrm{t}(\mathrm{s}-\mathrm{k}) \mathrm{t}_{\mathrm{s}-\mathrm{k}}=-\left(\mathrm{l}_{\mathrm{k}}-2\right) \mathrm{t}_{s-\mathrm{k}} \mathrm{t}\left(2 \leq i, j \leq \mathrm{l}_{\mathrm{k}}-2,0 \leq \mathrm{k} \leq \mathrm{s}\right) .
$$

Remark 3.3. Note that, if $\mathrm{s}=\mathrm{n}-1$, we have

$$
-n t \sum_{k=0}^{s} t_{s-k} M_{n}\left(x^{n-1}, x^{s-k}\right)=-n t \sum_{k=1}^{s} t_{s-k} M_{n}\left(x^{n-1}, x^{s-k}\right),
$$

Thus, when $i+j=l_{k}$, equation (3.4) should be modified by

$$
\hat{a}_{i j}(t)=-n t t_{s-k}+t(s-k) t_{s-k}=-\left(l_{k}-2\right) t_{s-k} t \quad\left(2 \leq i, j \leq l_{k}-2,1 \leq k \leq s\right) .
$$

We avoid this minor defect by considering that there is no entries satisfying $2 \leq i, j \leq l_{0}-2$ when $\mathrm{s}=\mathrm{n}-1$ since $\mathrm{l}_{0}-2=\mathrm{n}-\mathrm{s}=1$. 
Proposition 4. Put $l_{\mathrm{k}}=\mathrm{n}-\mathrm{s}+\mathrm{k}+2$. Then

$$
\begin{aligned}
& \hat{a}_{i j}(t)= \begin{cases}n & (i, j)=(1,1) \\
(s-k) t_{s-k} t & (i, j)=\left(1, l_{k}-1\right) \text { or }\left(l_{k}-1,1\right)(0 \leq k \leq s-1) \\
-\left(l_{k}-2\right) t_{s-k} t & i+j=l_{k}, 2 \leq i, j \leq l_{k}-2,(0 \leq k \leq s) \\
0 & \text { otherwise. }\end{cases} \\
& \tilde{a}_{i j}(t)= \begin{cases}b_{i-(n-s), j-(n-s)} t^{2} & n-s+1 \leq i, j \leq n \\
0 & \text { otherwise. }\end{cases}
\end{aligned}
$$

Proof. The statement for $\hat{a}_{i j}(t)$ has just been proved. For $\tilde{a}_{i j}(t)$, it is enough to see that we can denote

$$
\begin{aligned}
& M_{s}(g(x))=\sum_{\ell=0}^{s} \sum_{m=1}^{s} m t_{\ell} t_{m} M_{s}\left(x^{\ell}, x^{m-1}\right), \\
& M_{n}(g(x))=\sum_{\ell=0}^{s} \sum_{m=1}^{s} m t_{\ell} t_{m} M_{n}\left(x^{\ell}, x^{m-1}\right),
\end{aligned}
$$

that is, we can obtain $M_{n}(g(x))$ from $M_{s}(g(x))$ by just replacing $s$ with $n$ for all $M_{s}\left(x^{\ell}, x^{m}\right)$, which, by Lemma 3.1. means that $s \times s$ matrix $M_{s}(g(x))$ occupies the part $\left\{b_{i j}^{\dagger} \mid n-s+1 \leq i, j \leq n\right\}$ of the matrix $M_{n}(g(x))=\left(b_{i j}^{\dagger}\right)_{1 \leq i, j \leq n}$.

By Proposition 4, we can express the matrix $A(t)$ as follows;

$$
A(t)=\left[\begin{array}{cccc|cccc}
n & 0 & \ldots & 0 & s t_{s} t & (s-1) t_{s-1} t & \ldots & t_{1} t \\
0 & & & -(n-s) t_{s} t & -(n-s+1) t_{s-1} t & \ldots & -(n-1) t_{1} t & -n t_{0} t \\
\vdots & & . \cdot & . \cdot & & . \cdot & . \cdot & 0 \\
0 & -(n-s) t_{s} t & . \cdot & & & . \cdot & 0 & 0 \\
\hline s t_{s} t & -(n-s+1) t_{s-1} t & & & & & \\
(s-1) t_{s-1} t & \vdots & . \cdot & . \cdot & & & \\
\vdots & -(n-1) t_{1} t & . \cdot & 0 & & & & \\
t_{1} t & -n t_{0} t & 0 & 0 & & &
\end{array}\right] .
$$

Here, $C(t)=\left(c_{i j}(t)\right)_{1 \leq i, j \leq s}=C\left(t_{0}, \cdots, t_{s}, t\right)=\left(c_{i j}\left(t_{0}, \cdots, t_{s}, t\right)\right)_{1 \leq i, j \leq s}$ is an $s \times s$ symmetric matrix whose entries are of the form

$$
\begin{aligned}
c_{i j}\left(t_{0}, \cdots, t_{s}, t\right) & =b_{i j} t^{2}+\lambda_{i j} t \\
& =b_{i j}\left(t_{0}, \cdots, t_{s}\right) t^{2}+\lambda_{i j}\left(t_{0}, \cdots, t_{s}\right) t \quad\left(\lambda_{i j}=\lambda_{i j}\left(t_{0}, \cdots, t_{s}\right) \in E_{1}\right) .
\end{aligned}
$$

Next, let $A(t)_{1}=\left(a_{i j}(t)_{1}\right)_{1 \leq i, j \leq n}=A\left(t_{0}, \cdots, t_{s}, t\right)_{1}=\left(a_{i j}\left(t_{0}, \cdots, t_{s}, t\right)_{1}\right)_{1 \leq i, j \leq n}$ be the $n \times n$ symmetric matrix obtained from $A(t)$ by multiplying the first row and the first column by $1 / \sqrt{n}$ and then sweeping out the entries of the first row and the first column by the $(1,1)$ entry 1 . Here, let $Q_{m}(k ; c)=\left(q_{i j}\right)_{1 \leq i, j \leq m}$ and $R_{m}(k, l ; c)=\left(r_{i j}\right)_{1 \leq i, j \leq m}$ be $m \times m$ elementary matrices such that 


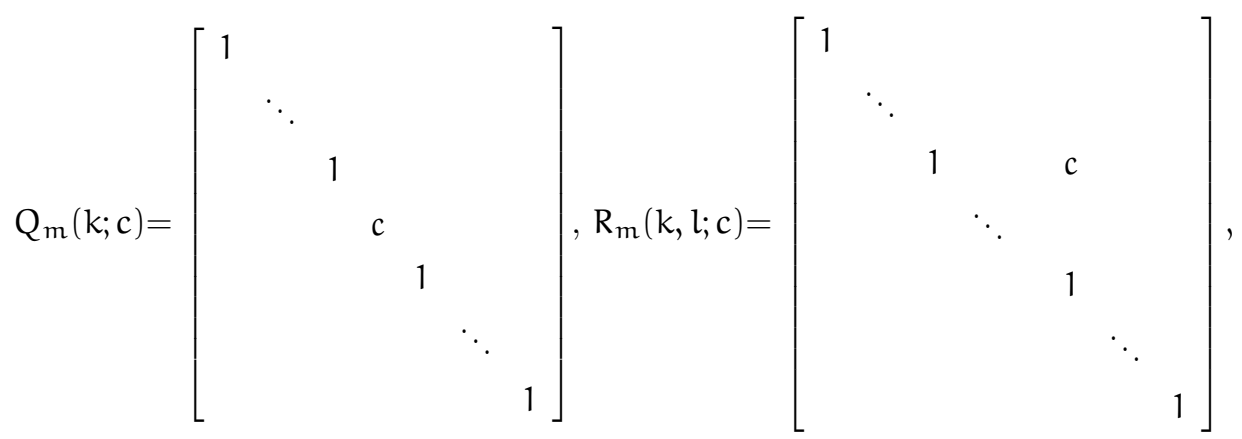

where $q_{k k}=c$ and $r_{k l}=c$. Moreover, for any $m \times m$ matrices $M_{1}, M_{2}, \cdots, M_{l}$, put $\prod_{k=1}^{l} M_{k}=$ $M_{1} M_{2} \cdots M_{l}$. Then, we have $A(t)_{1}={ }^{t} S(t)_{1} A(t) S(t)_{1}$, where

$$
S(t)_{1}=Q_{n}(1 ; 1 / \sqrt{n}) \prod_{k=0}^{s-1} R_{n}\left(1, l_{k}-1 ;-a_{1, l_{k}-1}(t) / \sqrt{n}\right) .
$$

The matrix $A(t){ }_{1}$ can be expressed as follows;

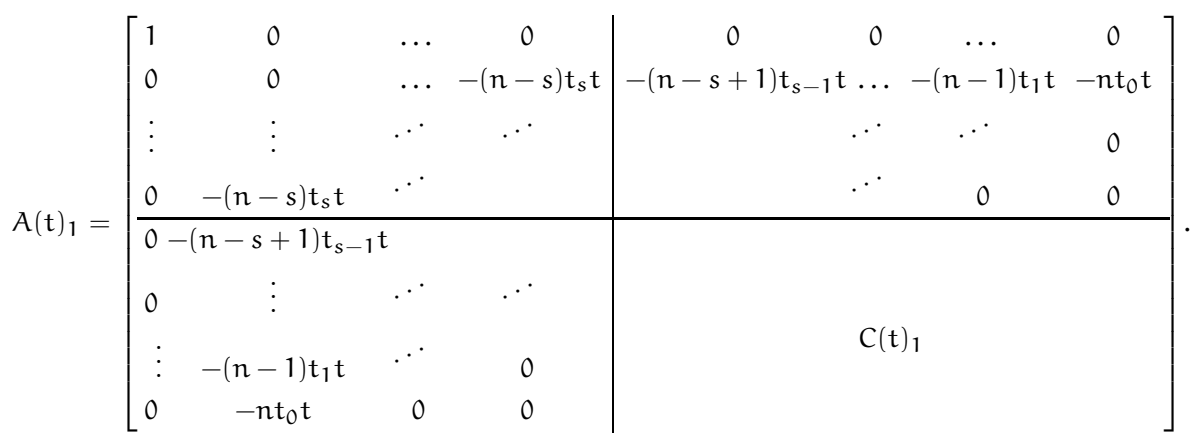

Here, $C(t)_{1}=\left(c_{i j}(t)_{1}\right)_{1 \leq i, j \leq s}=C\left(t_{0}, \cdots, t_{s}, t\right)_{1}=\left(c_{i j}\left(t_{0}, \cdots, t_{s}, t\right)_{1}\right)_{1 \leq i, j \leq s}$ is an $s \times s$ symmetric matrix whose entries are of the form

$$
c_{i j}\left(t_{0}, \cdots, t_{s}, t\right)_{1}=\bar{b}_{i j}\left(t_{0}, \cdots, t_{s}\right) t^{2}+\lambda_{i j}\left(t_{0}, \cdots, t_{s}\right) t \quad\left(\bar{b}_{i j}\left(t_{0}, \cdots, t_{s}\right) \in E_{1}\right),
$$

where

$$
\bar{b}_{i j}\left(t_{0}, \cdots, t_{s}\right)=b_{i j}\left(t_{0}, \cdots, t_{s}\right)-\frac{(s-i+1)(s-j+1)}{n} t_{s-i+1} t_{s-j+1}
$$

for any $i, j(1 \leq i, j \leq s)$. We put $\bar{b}_{i j}\left(t_{0}, \cdots, t_{s}\right)=\bar{b}_{i j}$ and $\bar{B}=\left(\bar{b}_{i j}\right)_{1 \leq i, j \leq s}$.

\subsection{Some results for the Bezoutian of $f_{r}(t ; x)$}

Let $r=\left(r_{0}, \cdots, r_{s}\right) \in \mathbb{R}^{s+1}$ be a vector as in Theorem 3.2 We put

$$
\begin{aligned}
& A_{r}(t)=\left(a_{i j}^{(r)}(t)\right)_{1 \leq i, j \leq n}=A\left(r_{0}, \cdots, r_{s}, t\right) \in \operatorname{Sym}_{n}(\mathbb{R}(t)), \\
& B_{r}=\left(b_{i j}^{(r)}\right)_{1 \leq i, j \leq s}=B\left(r_{0}, \cdots, r_{s}\right) \in \operatorname{Sym}_{s}(\mathbb{R})
\end{aligned}
$$


and $B_{r}(t)=t^{2} B_{r}$. Let us also put $A_{r}(t)_{1}=A\left(r_{0}, \cdots, r_{s}, t\right)_{1}$. By equation (3.6), the matrix $A_{r}(t)_{1}$ can be expressed as follows;

$$
A_{r}(t)_{1}=\left[\begin{array}{cccc|cccc}
1 & 0 & \ldots & 0 & 0 & 0 & \ldots & 0 \\
0 & 0 & \ldots & -(n-s) r_{s} t & -(n-s+1) r_{s-1} t & \ldots & -(n-1) r_{1} t & -n r_{0} t \\
\vdots & \vdots & . . & . . & \ldots & . . & 0 \\
0 & -(n-s) r_{s} t & . . & & . . & 0 & 0 \\
\hline 0-(n-s+1) r_{s-1} t & & \ldots & & \\
0 & \vdots & . . & . . & & & \\
\vdots & -(n-1) r_{1} t & . . & 0 & C_{r}(t) & \\
0 & -n r_{0} t & 0 & 0 & &
\end{array}\right] .
$$

Here, $\mathrm{C}_{\mathrm{r}}(\mathrm{t})_{1}=\left(\mathrm{c}_{i j}^{(\mathrm{r})}(\mathrm{t})_{1}\right)_{1 \leq i, j \leq s}=\mathrm{C}\left(\mathrm{r}_{0}, \cdots, \mathrm{r}_{\mathrm{s}}, \mathrm{t}\right)_{1}$ and

$$
c_{i j}^{(r)}(t)_{1}=\bar{b}_{i j}\left(r_{0}, \cdots, r_{s}\right) t^{2}+\lambda_{i j}\left(r_{0}, \cdots, r_{s}\right) t \quad\left(\bar{b}_{i j}\left(r_{0}, \cdots, r_{s}\right), \lambda_{i j}\left(r_{0}, \cdots, r_{s}\right) \in \mathbb{R}\right) .
$$

Note that, by equation (3.7), we have

$$
\bar{b}_{i j}\left(r_{0}, \cdots, r_{s}\right)=b_{i j}^{(r)}-\frac{(s-i+1)(s-j+1)}{n} r_{s-i+1} r_{s-j+1} \quad(1 \leq i, j \leq s) .
$$

To ease notation, we put $\overline{\mathrm{b}}_{i j}\left(\mathrm{r}_{0}, \cdots, \mathrm{r}_{\mathrm{s}}\right)=\overline{\mathrm{b}}_{\mathrm{ij}}^{(\mathrm{r})}$ and $\overline{\mathrm{B}}_{\mathrm{r}}=\left(\overline{\mathrm{b}}_{\mathbf{i j}}^{(\mathrm{r})}\right)_{1 \leq i, j \leq s}$.

In particular, since

$$
\begin{aligned}
M_{s}\left(g_{r}\right) & =M_{s}\left(r_{s} x^{s}, \sum_{k=0}^{s-1}(s-k) r_{s-k} x^{s-k-1}\right)+M_{s}\left(\sum_{k=1}^{s} r_{s-k} x^{s-k}, g_{r}^{\prime}\right) \\
& =\sum_{k=0}^{s-1}(s-k) r_{s} r_{s-k} M_{s}\left(\chi^{s}, \chi^{s-k-1}\right)+M_{s}\left(\sum_{k=1}^{s} r_{s-k} x^{s-k}, g_{r}^{\prime}\right)
\end{aligned}
$$

we have

$$
b_{1, k+1}^{(r)}=b_{k+1,1}^{(r)}=(s-k) r_{s} r_{s-k}(0 \leq k \leq s-1)
$$

by Lemma 3.1 and hence

$$
\begin{aligned}
\bar{b}_{1 j}^{(r)} & =(s-j+1) r_{s} r_{s-j+1}-\frac{s(s-j+1)}{n} r_{s} r_{s-j+1} \\
& =(s-j+1)\left(1-\frac{s}{n}\right) r_{s} r_{s-j+1}(1 \leq j \leq s) .
\end{aligned}
$$

Lemma 3.2. Put $\overline{\mathrm{B}}_{\mathrm{r}}(\mathrm{t})=\mathrm{t}^{2} \overline{\mathrm{B}}_{\mathrm{r}}$. Then, $\mathrm{B}_{\mathrm{r}}(\xi)$ and $\overline{\mathrm{B}}_{\mathrm{r}}(\xi)$ are equivalent over $\mathbb{R}$ for any real number $\xi$ and we have $\sigma\left(\overline{\mathrm{B}}_{\mathrm{r}}(\xi)\right)=\mathrm{N}_{\mathrm{g}_{\mathrm{r}}}$ for any non-zero real number $\xi$.

Proof. Let us denote by $\mathrm{B}_{r}^{*}=\left(\mathrm{b}_{i j}^{(\mathrm{r}, *)}\right)_{1 \leq i, j \leq s}\left(\overline{\mathrm{B}}_{\mathrm{r}}^{*}=\left(\overline{\mathrm{b}}_{i j}^{(\mathrm{r}, *)}\right)_{1 \leq i, j \leq s}\right)$ the matrix obtained from $\mathrm{B}_{\mathrm{r}}\left(\overline{\mathrm{B}}_{\mathrm{r}}\right)$ by multiplying the first row and the first column by $1 / \pm \sqrt{\mathbf{b}_{11}^{(\mathrm{r})}}\left(1 / \pm \sqrt{\overline{\mathrm{b}}_{11}^{(\mathrm{r})}}\right)$ (the sign 
before $\sqrt{b_{11}^{(r)}}\left(\sqrt{\bar{b}_{11}^{(r)}}\right)$ are the same as the sign of $r_{s}$; see the definition of $d(\bar{d})$ below $)$ and then sweeping out the entries of the first row and the first column by the $(1,1)$ entry 1 . Since $b_{11}=s r_{s}^{2}$ $(>0)$ and $\bar{b}_{11}=s(1-s / n) r_{s}^{2}(>0)$ by (3.8) and (3.9), we have

$$
\mathrm{B}_{\mathrm{r}}^{*}={ }^{\mathrm{t}} \mathrm{TB}_{\mathrm{r}} \mathrm{T}, \overline{\mathrm{B}}_{\mathrm{r}}^{*}={ }^{\mathrm{t}} \overline{\mathrm{T}}_{\mathrm{r}} \overline{\mathrm{T}}
$$

where

$$
\begin{aligned}
& T=Q_{s}(1 ; 1 / d) \prod_{k=2}^{s} R_{s}\left(1, k ;-b_{1 k}^{(r)} / d\right)\left(d=\sqrt{s} \cdot r_{s}\right) \\
& \bar{T}=Q_{s}(1 ; 1 / \bar{d}) \prod_{k=2}^{s} R_{s}\left(1, k ;-\bar{b}_{1 k}^{(r)} / \bar{d}\right)\left(\bar{d}=\sqrt{s(1-s / n)} \cdot r_{s}\right)
\end{aligned}
$$

Note that in [13, Lemma 3.3], we have proved $b_{i j}^{(r, *)}=\bar{b}_{i j}^{(r, *)}(1 \leq i, j \leq s)$ and hence $t^{2} B_{r}^{*}=t^{2} \bar{B}_{r}^{*}$, which, by (3.10), implies that symmetric matrices $B_{r}(\xi)$ and $\bar{B}_{r}(\xi)$ are equivalent over $\mathbb{R}$ for any real number $\xi$. Then, since $N_{g_{r}}=\sigma\left(B_{r}\right)=\sigma\left(B_{r}(\xi)\right)$ for any $\xi \in \mathbb{R} \backslash\{0\}$, the latter half of the statement have also been proved.

\subsection{Nonvanishingness of some coefficients}

In this subsection, we prove the next lemma.

Lemma 3.3. Let

$$
\Phi(x)=\Phi\left(t_{0}, \cdots, t_{s} ; x\right)=\sum_{k=0}^{s} h_{s-k}\left(t_{0}, \cdots, t_{s}\right) x^{s-k} \in E_{1}[x]
$$

be the characteristic polynomial of $\overline{\mathrm{B}}$. Then, $\mathrm{h}_{\mathrm{s}-\mathrm{k}}\left(\mathrm{t}_{0}, \cdots, \mathrm{t}_{\mathrm{s}}\right)$ is a non-zero polynomial in $\mathrm{E}_{1}$ for any $\mathrm{k}(1 \leq \mathrm{k} \leq \mathrm{s})$.

Proof. Lemma 3.3 is clear for $\mathrm{s}=1$, since we have

$$
B=M_{1}\left(t_{1} x+t_{0}\right)=\left[t_{1}^{2}\right]
$$

and hence, by equation (3.7),

$$
\bar{B}=\left[t_{1}^{2}-\frac{1}{n} t_{1}^{2}\right]=\left[\frac{n-1}{n} t_{1}^{2}\right] .
$$

Next, suppose $s \geq 2$. Then, by equation (3.7) and the definition of the Bezoutian, we have $h_{s-k}\left(t_{0}, \cdots, t_{s}\right) \in \mathbb{R}\left[t_{0}, \cdots, t_{s}\right]$ for any $k(1 \leq k \leq s)$. Thus, we have only to prove that $h_{s-k}\left(t_{0}, \cdots, t_{s}\right) \neq 0$ for any $k(1 \leq k \leq s)$, which is clear from the next Lemma 3.4

Lemma 3.4. Suppose $\mathrm{s} \geq 2$ and put $\mathrm{u}_{0}=\mathrm{u}_{\mathrm{s}}=1, \mathrm{u}_{1}=\mathrm{t}_{1}$ and $\mathfrak{u}_{\mathrm{k}}=0(2 \leq \mathrm{k} \leq \mathrm{s}-1)$. Then, $\mathrm{h}_{\mathrm{s}-\mathrm{k}}\left(\mathrm{u}_{0}, \cdots, \mathrm{u}_{\mathrm{s}}\right)$ is a non-constant polynomial in $\mathbb{R}\left(\mathrm{t}_{1}\right)$ for any $\mathrm{k}(1 \leq \mathrm{k} \leq \mathrm{s})$, i.e., $h_{s-k}\left(u_{0}, \cdots, u_{s}\right) \in \mathbb{R}\left[t_{1}\right] \backslash \mathbb{R}(1 \leq k \leq s)$. 
To prove lemma 3.4, let us put $\mathfrak{u}=\left(\mathfrak{u}_{0}, \cdots, \mathfrak{u}_{\mathrm{s}}\right)$ and

$$
\begin{aligned}
& g_{u}(x)=g\left(u_{0}, \cdots, u_{s} ; x\right)=x^{s}+t_{1} x+1 \in \mathbb{R}\left(t_{1}\right)[x], \\
& f_{u}(t ; x)=x^{n}+t_{\mathfrak{u}}(x) \in \mathbb{R}\left(t_{1}, t\right)[x](n>s), \\
& A_{u}(t)=\left(a_{i j}^{(u)}(t)\right)_{1 \leq i, j \leq n}=A\left(u_{0}, \cdots, u_{s}, t\right) \in \operatorname{Sym}_{n}\left(\mathbb{R}\left(t_{1}, t\right)\right), \\
& B_{u}=\left(b_{i j}^{(u)}\right)_{1 \leq i, j \leq s}=B\left(t_{0}, \cdots, u_{s}\right) \in \operatorname{Sym}_{s}\left(\mathbb{R}\left(t_{1}\right)\right), B_{u}(t)=t^{2} B_{u} .
\end{aligned}
$$

Then, by equation (3.5), we have

$$
A_{\mathfrak{u}}(t)=\left[\begin{array}{cccc|cccc}
n & 0 & \ldots & 0 & s t & 0 & \ldots & t_{1} t \\
0 & & & -(n-s) t & 0 & \ldots & -(n-1) t_{1} t & -n t \\
\vdots & & . . & . \cdot & & . . & . . & 0 \\
0 & -(n-s) t & . . & & & . . & 0 & 0 \\
\hline s t & 0 & & & & & \\
0 & \vdots & . . & . . & & & \\
\vdots & -(n-1) t_{1} t & . . & 0 & & & \\
t_{1} t & -n t & 0 & 0 & & &
\end{array}\right],
$$

where $\mathrm{C}_{\mathfrak{u}}(\mathrm{t})=\left(\mathrm{c}_{\mathrm{ij}}^{(\mathfrak{u})}(\mathrm{t})\right)_{1 \leq i, j \leq s}=\mathrm{C}\left(\mathrm{u}_{0}, \cdots, \mathrm{u}_{\mathrm{s}}, \mathrm{t}\right)$ and

$$
c_{i j}^{(u)}(t)=b_{i j}\left(u_{0}, \cdots, u_{s}\right) t^{2}+\lambda_{i j}\left(u_{0}, \cdots, u_{s}\right) t \quad\left(\lambda_{i j}\left(u_{0}, \cdots, u_{s}\right) \in \mathbb{R}\left(t_{1}\right)\right) .
$$

Moreover, by equation (3.6), we also have

$$
A_{\mathfrak{u}}(\mathrm{t})_{1}=\left[\begin{array}{cccc|cccc}
1 & 0 & \ldots & 0 & 0 & 0 & \ldots & 0 \\
0 & 0 & \ldots & -(n-s) t & 0 & \ldots & -(n-1) t_{1} t & -n t \\
\vdots & \vdots & . \cdot & . \cdot & & . . & . . & 0 \\
0 & -(n-s) t & . \cdot & & & . . & 0 & 0 \\
\hline 0 & 0 & & & & & \\
0 & \vdots & . \cdot & . . & & & \\
\vdots & -(n-1) t_{1} t & . \cdot & 0 & & & & \\
0 & -n t & 0 & 0 & & &
\end{array}\right] .
$$

Here, $\mathrm{C}_{\mathfrak{u}}(\mathrm{t})_{1}=\left(\mathrm{c}_{\mathrm{ij}}^{(\mathrm{u})}(\mathrm{t})_{1}\right)_{1 \leq i, j \leq s}=\mathrm{C}\left(\mathrm{u}_{0}, \cdots, \mathrm{u}_{\mathrm{s}}, \mathrm{t}\right)_{1}$ and

$$
c_{i j}^{(u)}(t)_{1}=\bar{b}_{i j}\left(u_{0}, \cdots, u_{s}\right) t^{2}+\lambda_{i j}\left(u_{0}, \cdots, u_{s}\right) t \quad\left(\bar{b}_{i j}\left(u_{0}, \cdots, u_{s}\right) \in \mathbb{R}\right) .
$$

Note that, by equation (3.7), we have

$$
\bar{b}_{i j}^{(u)}= \begin{cases}b_{11}^{(u)}-\left(s^{2} / n\right) & (i, j)=(1,1) \\ b_{1 s}^{(u)}-(s / n) t_{1} & (i, j)=(1, s) \text { or }(s, 1) \\ b_{s s}^{(u)}-(1 / n) t_{1}^{2} & (i, j)=(s, s) \\ b_{i j}^{(u)} & \text { otherwise. }\end{cases}
$$


Let us put $\bar{B}_{\mathfrak{u}}=\left(\bar{b}_{i j}^{(\mathfrak{u})}\right)_{1 \leq i, j \leq s}$ and $\bar{B}_{\mathfrak{u}}(t)=t^{2} \bar{B}_{\mathfrak{u}}$. Then, since

$$
\begin{aligned}
M_{s}\left(g_{u}\right)= & M_{s}\left(x^{s}+t_{1} x+1, s x^{s-1}+t_{1}\right) \\
= & s M_{s}\left(x^{s}, x^{s-1}\right)+t_{1} M_{s}\left(x^{s}, 1\right)-s t_{1} M_{s}\left(x^{s-1}, x\right)-s M_{s}\left(x^{s-1}, 1\right) \\
& +t_{1}^{2} M_{s}(x, 1)+t_{1} M_{s}(1,1)
\end{aligned}
$$

we have

(a) if $s=2$,

$$
B_{u}=\left[\begin{array}{cc}
2 & t_{1} \\
t_{1} & t_{1}^{2}-2
\end{array}\right]
$$

(b) if $s \geq 3$,

$$
b_{i j}^{(\mathfrak{u})}= \begin{cases}s & (i, j)=(1,1) \\ t_{1} & (i, j)=(1, s) \text { or }(s, 1) \\ (1-s) t_{1} & i+j=s+1,2 \leq i, j \leq s-1 \\ -s & i+j=s+2 \\ t_{1}^{2} & (i, j)=(s, s), \\ 0 & \text { otherwise, }\end{cases}
$$

which, by equation (3.12), implies

$\left(a^{\prime}\right)$ if $s=2$,

$$
\bar{B}_{u}=\left[\begin{array}{cc}
2(n-2) / n & (n-2) t_{1} / n \\
(n-2) t_{1} / n & (n-1) t_{1}^{2} / n-2
\end{array}\right],
$$

$\left(b^{\prime}\right)$ if $s \geq 3$

$$
\bar{b}_{i j}^{(u)}= \begin{cases}s(n-s) / n & (i, j)=(1,1) \\ (n-s) t_{1} / n & (i, j)=(1, s) \text { or }(s, 1) \\ (1-s) t_{1} & i+j=s+1,2 \leq i, j \leq s-1 \\ -s & i+j=s+2 \\ (n-1) t_{1}^{2} / n & (i, j)=(s, s), \\ 0 & \text { otherwise. }\end{cases}
$$

Therefore, if $s \geq 3$, the matrix $\bar{B}_{\mathfrak{u}}=\left(\bar{b}_{i j}^{(\mathfrak{u})}\right)_{1 \leq i, j \leq s}$ has the expression of the form

$$
\left[\begin{array}{ccccccc}
s(\mathrm{n}-\mathrm{s}) / \mathrm{n} & 0 & 0 & 0 & \ldots & 0 & (\mathrm{n}-\mathrm{s}) \mathrm{t}_{1} / \mathrm{n} \\
0 & 0 & 0 & \ldots & 0 & (1-\mathrm{s}) \mathrm{t}_{1} & -\mathrm{s} \\
0 & 0 & & . . & (1-\mathrm{s}) \mathrm{t}_{1} & -\mathrm{s} & 0 \\
0 & \vdots & . . & . . & . . & . . & \vdots \\
\vdots & 0 & (1-\mathrm{s}) \mathrm{t}_{1} & . . & . . & & 0 \\
0 & (1-\mathrm{s}) \mathrm{t}_{1} & -\mathrm{s} & . \cdot & & & 0 \\
(\mathrm{n}-\mathrm{s}) \mathrm{t}_{1} / \mathrm{n} & -\mathrm{s} & 0 & \ldots & 0 & 0 & (\mathrm{n}-1) \mathrm{t}_{1}^{2} / \mathrm{n}
\end{array}\right]
$$


Here, let us denote by

$$
\Phi_{\mathfrak{u}}(x)=\sum_{k=0}^{s} h_{s-k}^{(u)} x^{s-k}=\Phi\left(u_{0}, \cdots, u_{s} ; x\right)\left(=\sum_{k=0}^{s} h_{s-k}\left(u_{0}, \cdots, u_{s}\right) x^{s-k}\right)
$$

the characteristic polynomial of $\bar{B}_{\mathfrak{u}}$. Note that since we have $h_{s-k}^{(u)} \in \mathbb{R}\left[t_{1}\right]$ by the proof of Lemma 3.3. we have only to prove $h_{s-k}^{(u)}$ is non-constant for any $k(1 \leq k \leq s)$.

By the above expression of $\bar{B}_{\mathfrak{u}}$, we have

$\left(a^{\prime \prime}\right)$ if $s=2$,

$$
\Phi_{u}(x)=x^{2}-\frac{(n-1) t_{1}^{2}-4}{n} x+\frac{(n-2) t_{1}^{2}-4 n+8}{n}
$$

$\left(b^{\prime \prime}\right)$ if $s \geq 3$,

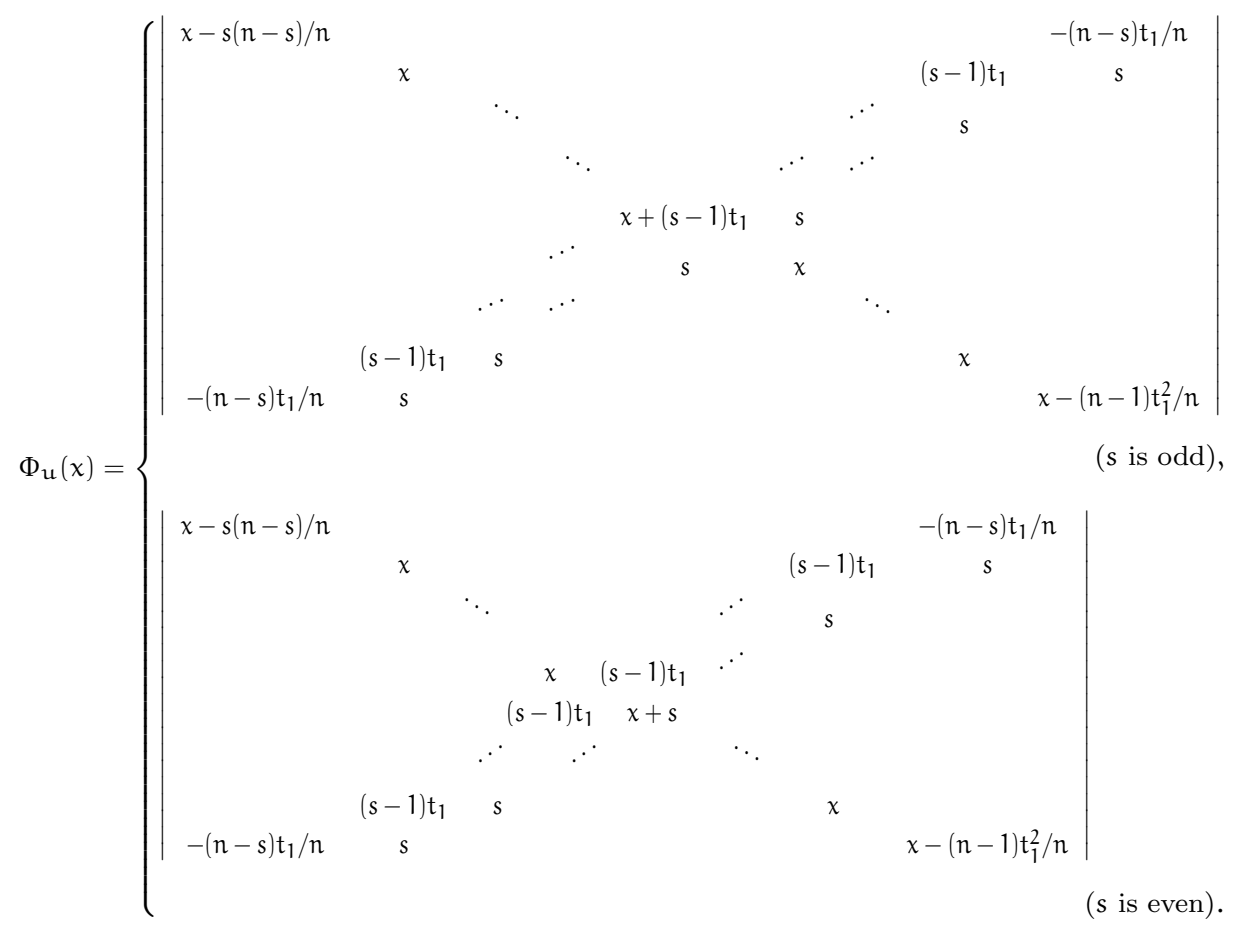

Example 3.1. (1) Put $\mathrm{s}=7$ and $\mathrm{n}=10$. Then, we have

$$
g_{\mathfrak{u}}(x)=x^{7}+t_{1} x+1, \quad f_{\mathfrak{u}}(t ; x)=x^{10}+t\left(x^{7}+t_{1} x+1\right),
$$




$$
\begin{aligned}
\Phi_{u}(x)= & \left|\begin{array}{ccccccc}
x-21 / 10 & 0 & 0 & 0 & 0 & 0 & -3 t_{1} / 10 \\
0 & x & 0 & 0 & 0 & 6 t_{1} & 7 \\
0 & 0 & x & 0 & 6 t_{1} & 7 & 0 \\
0 & 0 & 0 & x+6 t_{1} & 7 & 0 & 0 \\
0 & 0 & 6 t_{1} & 7 & x & 0 & 0 \\
0 & 6 t_{1} & 7 & 0 & 0 & x & 0 \\
-3 t_{1} / 10 & 7 & 0 & 0 & 0 & 0 & x-9 t_{1}^{2} / 10
\end{array}\right| \\
= & x^{7}+\left(-\frac{9}{10} t_{1}^{2}+6 t_{1}-\frac{21}{10}\right) x^{6}+\left(-\frac{27}{5} t_{1}^{3}-\frac{351}{5} t_{1}^{2}-\frac{63}{5} t_{1}-147\right) x^{5} \\
& +\left(\frac{324}{5} t_{1}^{4}-\frac{2106}{5} t_{1}^{3}+\frac{1197}{5} t_{1}^{2}-588 t_{1}+\frac{3087}{10}\right) x^{4} \\
& +\left(\frac{1944}{5} t_{1}^{5}+\frac{5832}{5} t_{1}^{4}+\frac{5859}{5} t_{1}^{3}+\frac{16758}{5} t_{1}^{2}+\frac{6174}{5} t_{1}+7203\right) x^{3} \\
& +\left(-\frac{5832}{5} t_{1}^{6}+\frac{34992}{5} t_{1}^{5}-\frac{21546}{5} t_{1}^{4}+\frac{50274}{5} t_{1}^{3}-\frac{95697}{10} t_{1}^{2}+14406 t_{1}-\frac{151263}{10}\right) x^{2} \\
+ & \left(-\frac{34992}{5} t_{1}^{7}+\frac{11664}{5} t_{1}^{6}-\frac{81648}{5} t_{1}^{5}+\frac{15876}{5} t_{1}^{4}-\frac{111132}{5} t_{1}^{3}+\frac{21609}{5} t_{1}^{2}-\frac{151263}{5} t_{1}\right. \\
- & 117649) x+\frac{69984}{5} t_{1}^{7}+\frac{2470629}{10} .
\end{aligned}
$$

(2) Put $\mathrm{s}=8$ and $\mathrm{n}=12$. Then, we have

$$
g_{u}(x)=x^{8}+t_{1} x+1, f_{u}(t ; x)=x^{12}+t\left(x^{8}+t_{1} x+1\right)
$$

and

$$
\begin{aligned}
& =\left|\begin{array}{cccccccc}
x-8 / 3 & 0 & 0 & 0 & 0 & 0 & 0 & -t_{1} / 3 \\
0 & x & 0 & 0 & 0 & 0 & 7 t_{1} & 8 \\
0 & 0 & x & 0 & 0 & 7 t_{1} & 8 & 0 \\
0 & 0 & 0 & x & 7 t_{1} & 8 & 0 & 0 \\
0 & 0 & 0 & 7 t_{1} & x+8 & 0 & 0 & 0 \\
0 & 0 & 7 t_{1} & 8 & 0 & x & 0 & 0 \\
0 & 7 t_{1} & 8 & 0 & 0 & 0 & x & 0 \\
-t_{1} / 3 & 8 & 0 & 0 & 0 & 0 & 0 & x-11 t_{1}^{2} / 12
\end{array}\right| \\
= & x^{8}+\left(-\frac{11}{12} t_{1}^{2}+\frac{16}{3}\right) x^{7}+\left(-152 t_{1}^{2}-\frac{640}{3}\right) x^{6}+\left(\frac{539}{4} t_{1}^{4}-256 t_{1}^{2}-1024\right) x^{5} \\
& +\left(\frac{22736}{3} t_{1}^{4}+\frac{45824}{3} t_{1}^{2}+16384\right) x^{4}+\left(-\frac{26411}{4} t_{1}^{6}-\frac{22736}{3} t_{1}^{4}+\frac{31744}{3} t_{1}^{2}+65536\right) x^{3} \\
& +\left(-\frac{355348}{3} t_{1}^{6}-213248 t_{1}^{4}-\frac{1064960}{3} t_{1}^{2}-524288\right) x^{2}+\left(\frac{1294139}{12} t_{1}^{8}+\frac{1075648}{3} t_{1}^{6}\right. \\
& \left.+\frac{1404928}{3} t_{1}^{4}+\frac{1835008}{3} t_{1}^{2}-\frac{4194304}{3}\right) x-\frac{823543}{3} t_{1}^{8}+\frac{16777216}{3} .
\end{aligned}
$$

Proof of Lemma 3.4. To prove Lemma 3.4, it is enough to prove $\operatorname{deg} h_{s-k}^{(u)} \geq 1$ for any $k(1 \leq k \leq s)$. This is clear for $s=2$ by $\left(a^{\prime \prime}\right)$ and we suppose $s \geq 3$ hereafter. To prove $\operatorname{deg} h_{s-k}^{(u)} \geq 1(1 \leq k \leq s)$, 
let us compute the leading term of $h_{s-k}^{(\mathfrak{u})}\left(\in \mathbb{R}\left[\mathfrak{t}_{1}\right]\right)$. Then, since $h_{s-k}^{(\mathfrak{u})}$ is the coefficient of the term $h_{s-k}^{(u)} x^{s-k}$ of the characteristic polynomial $\Phi_{\mathfrak{u}}(x)$, we need to maximize the degree in $t_{1}$ when we take ' $s-k$ ' $x$ and the remaining $k$ elements from $\mathbb{R}\left[t_{1}\right]$.

(a) Suppose $s$ is odd. Let us divide the case into three other sub-cases.

(a1) Suppose $k$ is odd and $1 \leq k \leq s-2$.

In this case, the degree of the leading term of $h_{s-k}^{(\mathfrak{u})}$ is $k+1$. In fact, it is obtained by taking

(a11) $-(n-1) t_{1}^{2} / n$ from the $(s, s)$ entry $x-(n-1) t_{1}^{2} / n$,

(a12) ' $k-1$ ' $(s-1) t_{1}$ from entries of the form $(i, s+1-i)(2 \leq i \leq s-1)$.

First, suppose we take the $(s, s)$ entry $x-(n-1) t_{1}^{2} / n$ from the $s$-th row. Then we must take the $(1,1)$ entry from the first row. Next, let us proceed to the $(s-1)$-th row. If we take the $(s-1, s-1)$ entry $x$ from the $(s-1)$-th row, then we must also take $x$ from the second row, while if we take $(s-1) t_{1}$ from the $(s-1)$-th row, then we must also take $(s-1) t_{1}$ from the second row. The situation is the same for the $(s-2)$-th row, the $(s-3)$-th row ... and so on, which implies that $(s-1) t_{1}$ must occur in pair.

Hence, the leading term of $h_{s-k}^{(u)}$ is

$$
-\frac{n-1}{n} t_{1}^{2} \cdot\left(\begin{array}{l}
(s-3) / 2 \\
(k-1) / 2
\end{array}\right)\left\{(-1) \cdot(s-1)^{2} t_{1}^{2}\right\}^{(k-1) / 2} \quad\left(\left(\begin{array}{l}
n \\
0
\end{array}\right)=1(n \geq 0)\right)
$$

and the degree of this term is $k+1(\geq 2)$.

(a2) Suppose $k$ is odd and $k=s$.

If $k=s, h_{s-k}^{(u)}=h_{0}^{(u)}$ is the constant term of $\Phi_{\mathfrak{u}}(x)$. In this case, the degree of the leading term of $h_{0}^{(u)}$ is $s$. In fact, it is obtained by taking

(a21) $-(n-1) t_{1}^{2} / n$ from the $(s, s)$ entry $x-(n-1) t_{1}^{2} / n$,

(a22) If $s \geq 5(\Leftrightarrow(s, k) \neq(3,3))$, ' $(s-3) / 2$ ' pairs of $(s-1) t_{1}$ from entries of the form $(i, s+1-i)$ $(2 \leq i \leq(s-1) / 2,(s+3) / 2 \leq i \leq s-1)$,

(a23) $(s-1) t_{1}$ from the $((s+1) / 2,(s+1) / 2)$ entry $x+(s-1) t_{1}$,

(a24) $-s(n-s) / n$ from the $(1,1)$ entry $x-s(n-s) / n$

or by taking

(a25) all anti-diagonal entries. 
Therefore, the leading term of $h_{0}^{(u)}$ is

$$
\begin{aligned}
& -\frac{n-1}{n} t_{1}^{2} \cdot\left\{(-1) \cdot(s-1)^{2} t_{1}^{2}\right\}^{(s-3) / 2} \cdot(s-1) t_{1} \cdot\left(-\frac{s(n-s)}{n}\right) \\
& \quad+(-1) \cdot\left(-\frac{n-s}{n} t_{1}\right)^{2} \cdot\left\{(-1) \cdot(s-1)^{2} t_{1}^{2}\right\}^{(s-3) / 2} \cdot(s-1) t_{1} \\
& =\frac{(n-s)(s-1)}{n} \cdot(-1)^{(s-3) / 2}(s-1)^{s-2} t_{1}^{s} \\
& =(-1)^{(s-3) / 2} \frac{(n-s)(s-1)^{s-1}}{n} t_{1}^{s}
\end{aligned}
$$

for any $s(s \geq 3)$ and the degree of this term is $s$.

(a3) Suppose k is even.

In this case, we have $2 \leq k \leq s-1$ and the degree of the leading term of $h_{s-k}^{(u)}$ is $k+1$. In fact, it is obtained by taking

(a31) $-(n-1) t_{1}^{2} / n$ from the $(s, s)$ entry $x-(n-1) t_{1}^{2} / n$,

(a32) If $s \geq 5(\Leftrightarrow(s, k) \neq(3,2)), '(k-2) / 2$ ' pairs of $(s-1) t_{1}$ from entries of the form $(i, s+1-i)$ $(2 \leq i \leq(s-1) / 2,(s+3) / 2 \leq i \leq s-1)$,

(a33) $(s-1) t_{1}$ from the $((s+1) / 2,(s+1) / 2)$ entry $x+(s-1) t_{1}$.

Therefore, the leading term of $h_{s-k}^{(u)}$ is

$$
-\frac{n-1}{n} t_{1}^{2} \cdot\left(\begin{array}{l}
(s-3) / 2 \\
(k-2) / 2
\end{array}\right)\left\{(-1) \cdot(s-1)^{2} t_{1}^{2}\right\}^{(k-2) / 2} \cdot(s-1) t_{1}
$$

for any $s(s \geq 3)$ and the degree of this term is $k+1(\geq 3)$.

(b) Suppose $s$ is even $(s \geq 4)$. We also divide this case into three other sub-cases.

(b1) Suppose $k$ is odd.

In this case, we have $1 \leq k \leq s-1$ and the degree of the leading term of $h_{s-k}^{(u)}$ is $k+1$. In fact, it is obtained by taking

(b11) $-(n-1) t_{1}^{2} / n$ from the $(s, s)$ entry $x-(n-1) t_{1}^{2} / n$,

(b12) ' $(k-1) / 2$ ' pairs of $(s-1) t_{1}$ from entries of the form $(i, s+1-i)(2 \leq i \leq s-1)$.

Therefore, the leading term of $h_{s-k}^{(u)}$ is

$$
-\frac{n-1}{n} t_{1}^{2} \cdot\left(\begin{array}{l}
(s-2) / 2 \\
(k-1) / 2
\end{array}\right)\left\{(-1) \cdot(s-1)^{2} t_{1}^{2}\right\}^{(k-1) / 2}
$$

and the degree of this term is $k+1(\geq 2)$.

(b2) Suppose $k$ is even and $2 \leq k \leq s-2$.

In this case, the degree of the leading term of $h_{s-k}^{(u)}$ is $k$. In fact, it is obtained by taking 
(b21) $-(n-1) t_{1}^{2} / n$ from the $(s, s)$ entry $x-(n-1) t_{1}^{2} / n$,

(b22) ' $(k-2) / 2$ ' pairs of $(s-1) t_{1}$ from entries of the form $(i, s+1-i)(2 \leq i \leq s-1)$,

(b23) $-s(n-s) / n$ from the $(1,1)$ entry $x-s(n-s) / n$

or by taking

(b24) $-(n-1) t_{1}^{2} / n$ from the $(s, s)$ entry $x-(n-1) t_{1}^{2} / n$,

(b25) I f $s \geq 6(\Leftrightarrow(s, k) \neq(4.2))$, ' $(k-2) / 2$ ' pairs of $(s-1) t_{1}$ from entries of the form $(i, s+1-i)$ $(2 \leq i \leq(s-2) / 2,(s+4) / 2 \leq i \leq s-1)$,

(b26) $s$ from the $((s+2) / 2,(s+2) / 2)$ entry $x+s$

or by taking

(b27) ' $k / 2$ ' pairs of $(s-1) t_{1}$ from entries of the form $(i, s+1-i)(2 \leq i \leq s-1)$

or by taking

(b28) One pair of $-(n-s) t_{1} / n$ from the $(1, s)$ and the $(s, 1)$ entry,

(b29) ' $(k-2) / 2$ ' pairs of $(s-1) t_{1}$ from entries of the form $(i, s+1-i)(2 \leq i \leq s-1)$.

Here, note that if we take the $(s, 1)$ entry $-(n-s) t_{1} / n$ from the $s$-th row, we must also take the $(1, s)$ entry $-(n-s) t_{1} / n$ from the first row.

Therefore, the leading term of $h_{s-k}^{(u)}$ is

$$
\begin{aligned}
& -\frac{n-1}{n} t_{1}^{2} \cdot\left(\begin{array}{c}
(s-2) / 2 \\
(k-2) / 2
\end{array}\right)\left\{(-1) \cdot(s-1)^{2} t_{1}^{2}\right\}^{(k-2) / 2} \cdot\left(-\frac{s(n-s)}{n}\right) \\
& -\frac{n-1}{n} t_{1}^{2} \cdot\left(\begin{array}{c}
(s-4) / 2 \\
(k-2) / 2
\end{array}\right)\left\{(-1) \cdot(s-1)^{2} t_{1}^{2}\right\}^{(k-2) / 2} \cdot s+\left(\begin{array}{c}
(s-2) / 2 \\
k / 2
\end{array}\right)\left\{(-1) \cdot(s-1)^{2} t_{1}^{2}\right\}^{k / 2} \\
& +\left((-1) \cdot \frac{\{-(n-s)\}^{2}}{n^{2}} t_{1}^{2}\right) \cdot\left(\begin{array}{c}
(s-2) / 2 \\
(k-2) / 2
\end{array}\right)\left\{(-1) \cdot(s-1)^{2} t_{1}^{2}\right\}^{(k-2) / 2} \\
& =\left(\frac{s(n-s)(n-1)}{n^{2}}\left(\begin{array}{c}
(s-2) / 2 \\
(k-2) / 2
\end{array}\right)-\frac{s(n-1)}{n}\left(\begin{array}{c}
(s-4) / 2 \\
(k-2) / 2
\end{array}\right)\right. \\
& \left.\quad-(s-1)^{2}\left(\begin{array}{c}
(s-2) / 2 \\
k / 2
\end{array}\right)-\frac{(n-s)^{2}}{n^{2}}\left(\begin{array}{c}
(s-2) / 2 \\
(k-2) / 2
\end{array}\right)\right)\left\{(-1) \cdot(s-1)^{2} t_{1}^{2}\right\}^{(k-2) / 2} t_{1}^{2} .
\end{aligned}
$$

for any $s(s \geq 4)$. Then, since

$$
\left(\begin{array}{c}
(s-4) / 2 \\
(k-2) / 2
\end{array}\right)=\frac{s-k}{s-2}\left(\begin{array}{c}
(s-2) / 2 \\
(k-2) / 2
\end{array}\right),\left(\begin{array}{c}
(s-2) / 2 \\
k / 2
\end{array}\right)=\frac{s-k}{k}\left(\begin{array}{c}
(s-2) / 2 \\
(k-2) / 2
\end{array}\right),
$$

we have 


$$
\begin{aligned}
& \frac{s(n-s)(n-1)}{n^{2}}\left(\begin{array}{c}
(s-2) / 2 \\
(k-2) / 2
\end{array}\right)-\frac{s(n-1)}{n}\left(\begin{array}{c}
(s-4) / 2 \\
(k-2) / 2
\end{array}\right) \\
& -(s-1)^{2}\left(\begin{array}{c}
(s-2) / 2 \\
k / 2
\end{array}\right)-\frac{(n-s)^{2}}{n^{2}}\left(\begin{array}{c}
(s-2) / 2 \\
(k-2) / 2
\end{array}\right) \\
& =\left(\frac{s(n-s)(n-1)}{n^{2}}-\frac{s(s-k)(n-1)}{n(s-2)}-\frac{(s-1)^{2}(s-k)}{k}-\frac{(n-s)^{2}}{n^{2}}\right)\left(\begin{array}{l}
(s-2) / 2 \\
(k-2) / 2
\end{array}\right) \\
& =\frac{s\left\{\left(k\left(k+s^{2}-4 s+2\right)-s^{3}+4 s^{2}-5 s+2\right) n-k\left(k+s^{2}-4 s+2\right)\right\}}{n k(s-2)}\left(\begin{array}{l}
(s-2) / 2 \\
(k-2) / 2
\end{array}\right) .
\end{aligned}
$$

Hence, if the above value becomes zero, we have

$$
\left(k\left(k+s^{2}-4 s+2\right)-s^{3}+4 s^{2}-5 s+2\right) n-k\left(k+s^{2}-4 s+2\right)=0,
$$

which implies

$$
k\left(k+s^{2}-4 s+2\right)=0,-s^{3}+4 s^{2}-5 s+2=0
$$

or

$$
n=\frac{k\left(k+s^{2}-4 s+2\right)}{k\left(k+s^{2}-4 s+2\right)-s^{3}+4 s^{2}-5 s+2} .
$$

Here, (3.14) is impossible since $-s^{3}+4 s^{2}-5 s+2=-(s-1)^{2}(s-2)$ and $s \geq 4$. Also, (3.15) is impossible since, for any $s \geq 4$ and $2 \leq k \leq s-2$, we have

$$
k\left(k+s^{2}-4 s+2\right) \geq 2\left(2+s^{2}-4 s+2\right) \geq 2(s-2)^{2}>0
$$

and

$$
\begin{aligned}
& k\left(k+s^{2}-4 s+2\right)-s^{3}+4 s^{2}-5 s+2 \\
& \leq(s-2)\left\{(s-2)+s^{2}-4 s+2\right\}-s^{3}+4 s^{2}-5 s+2 \\
& =-s^{2}+s+2 \\
& =-(s+1)(s-2)<0,
\end{aligned}
$$

which implies $n<0$, a contradiction. Thus, the above value (3.13) is non-zero and the degree of the leading term of $h_{s-k}^{(u)}$ is $k$.

(b3) Suppose $k$ is even and $k=s$.

If $k=s, h_{s-k}^{(u)}=h_{0}^{(u)}$ is the constant term of $\Phi_{\mathfrak{u}}(x)$. In this case, the degree of the leading term of $h_{0}^{(u)}$ is s. In fact, it is obtained by taking

(b31) $-(n-1) t_{1}^{2} / n$ from the $(s, s)$ entry $x-(n-1) t_{1}^{2} / n$,

(b32) ' $(s-2) / 2$ ' pairs of $(s-1) t_{1}$ from entries of the form $(i, s+1-i)(2 \leq i \leq s-1)$,

(b33) $-s(n-s) / n$ from the $(1,1)$ entry $x-s(n-s) / n$

or by taking 
(b34) all anti-diagonal entries.

Therefore, the leading term of $h_{0}^{(u)}$ is

$$
\begin{aligned}
& -\frac{n-1}{n} t_{1}^{2} \cdot\left\{(-1) \cdot(s-1)^{2} t_{1}^{2}\right\}^{(s-2) / 2} \cdot\left(-\frac{s(n-s)}{n}\right) \\
& \quad+(-1) \cdot\left(-\frac{n-s}{n} t_{1}\right)^{2} \cdot\left\{(-1) \cdot(s-1)^{2} t_{1}^{2}\right\}^{(s-2) / 2} \\
& =(-1)^{(s-2) / 2} \frac{(n-s)(s-1)^{s-1}}{n} t_{1}^{s}
\end{aligned}
$$

and the degree of this term is $s(s \geq 4)$.

Lemma 3.5. Let $v=\left(v_{0}, \cdots, v_{s}\right) \in \mathbb{R}^{s+1}$ be a real vector and $\mathrm{n}(>\mathrm{s})$ be an integer. Put

$$
\mathrm{P}_{v}(\mathrm{t})=\operatorname{det} \mathrm{M}_{\mathrm{n}}\left(\mathrm{f}_{v}(\mathrm{t} ; \mathrm{x})\right)=\operatorname{det} \mathrm{M}_{\mathrm{n}}\left(\mathrm{f}^{(\mathrm{n})}\left(v_{0}, \cdots, v_{s}, \mathrm{t} ; \mathrm{x}\right)\right)
$$

and $\alpha_{v}=\max \left\{\alpha \in \mathbb{R} \mid \mathrm{P}_{v}(\alpha)=0\right\}$. If there exists a real number $\rho_{0}\left(>\alpha_{v}\right)$ such that $\mathrm{N}_{\mathrm{f}_{v}(\xi ; \mathrm{x})}=\gamma_{0}$ for any $\xi>\rho_{0}$, we have $\mathrm{N}_{\mathrm{f}_{v}(\xi ; \mathrm{x})}=\gamma_{0}$ for any $\xi>\alpha_{v}$.

Proof. Put $A_{v}(t)=M_{n}\left(f_{v}(t ; x)\right)$. Then, by Proposition 2, we have $\gamma_{0}=\sigma\left(A_{v}(\xi)\right)$ for any $\xi>\rho_{0}$. Let us also put

$$
R=\left\{\rho \in \mathbb{R} \mid \rho>\alpha_{v}, \sigma\left(A_{v}(\xi)\right)=\gamma_{0} \text { for any } \xi>\rho\right\} .
$$

Since R is a nonempty set $\left(\rho_{0} \in \mathrm{R}\right)$ having a lower bound $\alpha_{v}$, R has the infimum $\rho_{v} ; \rho_{v}=\inf \mathrm{R}$. Then, it is enough to prove $\rho_{v}=\alpha_{v}$. Here, suppose to the contrary that $\rho_{v}>\alpha_{v}$ and we denote by

$$
\Omega_{v}(t ; x)=\sum_{k=0}^{n} \omega_{k}(t) x^{k} \in \mathbb{R}(t)[x]
$$

the characteristic polynomial of $A_{v}(t)$. Note that $\omega_{k}(t) \in \mathbb{R}[t](0 \leq k \leq n)$ and for any $\xi>$ $\alpha_{v}, \Omega_{v}(\xi ; x)$ has $n$ non-zero real roots (counted with multiplicity) since $A_{v}(\xi)$ is symmetric and $\operatorname{det} A_{v}(\xi) \neq 0$. Then, by Proposition 3. there exists a positive real number $\delta$ such that $\rho_{v}-\delta>\alpha_{v}$ and for any $\xi \in\left[\rho_{v}-\delta, \rho_{v}+\delta\right], \Omega_{v}(\xi ; x)$ has the same number of positive and hence negative real roots with $\Omega_{v}\left(\rho_{v} ; x\right)$. On the other hand, since $\rho_{v}=\inf \mathrm{R}$, there exist real numbers $\xi_{+}$ $\left(\rho_{v}<\xi_{+}<\rho_{v}+\delta\right)$ and $\xi_{-}\left(\rho_{v}-\delta<\xi_{-}<\rho_{v}\right)$ such that $\sigma\left(A_{v}\left(\xi_{+}\right)\right) \neq \sigma\left(A_{v}\left(\xi_{-}\right)\right)$, which implies $\Omega_{v}\left(\xi_{+} ; x\right)$ and $\Omega_{v}\left(\xi_{-} ; x\right)$ have different number of positive and hence negative real roots. This is a contradiction and we have $\rho_{v}=\alpha_{v}$.

\subsection{Proof of Theorem 3.2}

Let $r=\left(r_{0}, \cdots, r_{s}\right) \in \mathbb{R}^{s+1}$ be the vector as in Theorem 3.2 and put

$$
n_{0}= \begin{cases}(n-s+1) / 2, & n-s-1: \text { even } \\ (n-s+2) / 2, & n-s-1: \text { odd } .\end{cases}
$$


When $n-s \geq 2$, we inductively define the matrix $A_{r}(t)_{k}=\left(a_{i j}^{(r)}(t)_{k}\right)_{1 \leq i, j \leq n}(2 \leq k \leq n-s)$ as the matrix obtained from $A_{r}(t)_{k-1}$ by sweeping out the entries of the $k$-th row (k-th column) by the $\left(k, l_{0}-k\right)$ entry $-(n-s) r_{s} t\left(\left(l_{0}-k, k\right)\right.$ entry $\left.-(n-s) r_{s} t\right)$. That is, we define $A_{r}(t)_{k}=$ ${ }^{t} S_{r}(t)_{k} A_{r}(t)_{k-1} S_{r}(t)_{k}$, where

$$
S_{r}(t)_{k}=\left\{\begin{array}{lr}
\prod_{m=l_{0}-k+1}^{n} R_{n}\left(l_{0}-k, m ;-\frac{a_{k m}^{(r)}(t)_{k-1}}{-(n-s) r_{s} t}\right) & \left(2 \leq k \leq n_{0}\right) \\
R_{n}\left(l_{0}-k, k ;-\frac{a_{k k}^{(r)}(t)_{k-1}}{-2(n-s) r_{s} t}\right) \prod_{m=k+1}^{n} R_{n}\left(l_{0}-k, m ;-\frac{a_{k m}^{(r)}(t)_{k-1}}{-(n-s) r_{s} t}\right) \\
\left(n_{0}<k \leq n-s\right) .
\end{array}\right.
$$

Then, if $n-s \geq 1$, we can express the matrix $A_{r}(t)_{n-s}$ as follows;

$$
A_{r}(t)_{n-s}=\left[\begin{array}{cccc|c}
1 & 0 & \ldots & 0 & \\
0 & 0 & \ldots & -(n-s) r_{s} t & O \\
\vdots & \vdots & . \cdot & 0 & \\
0 & -(n-s) r_{s} t & 0 & 0 & \\
\hline & & & & C_{r}(t)_{n-s}
\end{array}\right] .
$$

Note that $a_{k m}^{(r)}(t)_{k-1}$ and $a_{k k}^{(r)}(t)_{k-1}$ appearing in $S_{r}(t)_{k}$ are degree 1 monomials in $t$ and hence the numbers $-a_{k m}^{(r)}(t)_{k-1} /\left(-(n-s) r_{s} t\right),-a_{k k}^{(r)}(t)_{k-1} /\left(-2(n-s) r_{s} t\right)$ appearing in $S_{r}(t)_{k}$ are just real numbers. Therefore, the entries of the $s \times s$ symmetric matrix $C_{r}(t)_{n-s}=\left(c_{i j}^{(r)}(t)_{n-s}\right)_{1 \leq i, j \leq s}$ $(n-s \geq 1)$ are of the form

$$
c_{i j}^{(r)}(t)_{n-s}=\bar{b}_{i j}^{(r)} t^{2}+\bar{\lambda}_{i j}^{(r)} t \quad\left(\bar{\lambda}_{i j}^{(r)} \in \mathbb{R}\right) .
$$

Moreover, since the matrix

$$
\mathrm{D}_{r}(\mathrm{t})_{n-s}=\left[\begin{array}{cccc}
1 & 0 & \ldots & 0 \\
0 & 0 & \ldots & -(n-s) r_{s} t \\
\vdots & \vdots & . . & 0 \\
0 & -(n-s) r_{s} t & 0 & 0
\end{array}\right]
$$


is equivalent to the matrix

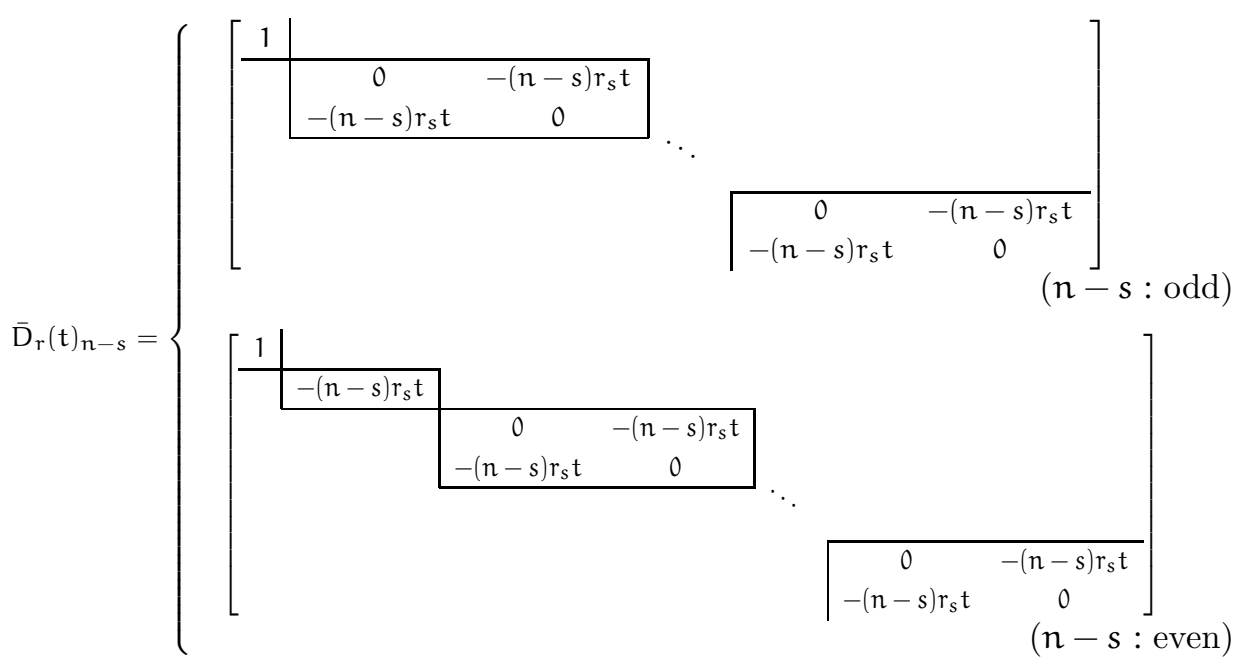

over $\mathbb{R}$, we have

$$
\sigma\left(D_{r}(\xi)_{n-s}\right)=\sigma\left(\bar{D}_{r}(\xi)_{n-s}\right)= \begin{cases}1 & n-s: \text { odd } \\ 0 & n-s: \text { even, } r_{s}>0 \\ 2 & n-s: \text { even, } r_{s}<0\end{cases}
$$

for any real number $\xi>\alpha_{r}(\geq 0)$. Here, note that since $P_{r}(0)=0$, we have $\alpha_{r} \geq 0$.

Next, let $\Phi_{r}(t ; x), \Psi_{r}(t ; x)$ be characteristic polynomials of $\bar{B}_{r}(t), C_{r}(t)_{n-s}$, respectively. Then, by equations (3.11) and (3.16), we have

$$
\begin{aligned}
& \Phi_{r}(t ; x)=x^{s}+h_{s-1}^{(r)} t^{2} \chi^{s-1}+\cdots+ h_{1}^{(r)} t^{2 s-2} x+h_{0}^{(r)} t^{2 s} \\
&\left(h_{s-k}^{(r)}=h_{s-k}\left(r_{0}, \cdots, r_{s}\right) \in \mathbb{R}(1 \leq k \leq s)\right), \\
& \Psi_{r}(t ; x)=x^{s}+\left(h_{s-1}^{(r)} t^{2}+\psi_{s-1}(t)\right) x^{s-1}+\cdots \\
&+\left(h_{1}^{(r)} t^{2 s-2}+\psi_{1}(t)\right) x+\left(h_{0}^{(r)} t^{2 s}+\psi_{0}(t)\right) \\
&\left(\psi_{0}(t), \cdots, \psi_{s-1}(t) \in \mathbb{R}[t], \operatorname{deg} \psi_{s-k}(t)<2 k(1 \leq k \leq s)\right) .
\end{aligned}
$$

Here, let us divide the proof into next two cases.

(i) The case $h_{0}^{(r)} h_{1}^{(r)} \cdots h_{s-1}^{(r)} \neq 0$.

In this case, we have

$$
\begin{aligned}
\Psi_{r}(t ; x)=x^{s}+h_{s-1}^{(r)} t^{2}(1 & \left.+\frac{\psi_{s-1}(t)}{h_{s-1}^{(r)} t^{2}}\right) x^{s-1}+\cdots \\
& +h_{1}^{(r)} t^{2 s-2}\left(1+\frac{\psi_{1}(t)}{h_{1}^{(r)} t^{2 s-2}}\right) x+h_{0}^{(r)} t^{2 s}\left(1+\frac{\psi_{0}(t)}{h_{0}^{(r)} t^{2 s}}\right)
\end{aligned}
$$


and $1+\psi_{s-k}(t) / h_{s-k}^{(r)} t^{2 k} \rightarrow 1(t \rightarrow \infty)$ for any $k(1 \leq k \leq s)$. Moreover, since $h_{0}^{(r)} h_{1}^{(r)} \cdots h_{s-1}^{(r)} \neq 0$, we have $h_{0}^{(r)} \neq 0$, which implies that for any non-zero real number $\xi, \Phi_{r}(\xi ; x)$ have s non-zero real roots (counted with multiplicity). Thus, there exists a real number $\rho_{0}\left(>\alpha_{r}\right)$ such that for any real number $\xi>\rho_{0}, \Psi_{r}(\xi ; x)$ have the same number of positive (hence also negative) real roots with $\Phi_{r}(\xi ; x)$ by Proposition 3, which implies $\sigma\left(C_{r}(\xi)_{n-s}\right)=\sigma\left(\bar{B}_{r}(\xi)\right)$ and hence $\sigma\left(C_{r}(\xi)_{n-s}\right)=$ $\mathrm{N}_{\mathrm{g}_{\mathrm{r}}}=\gamma\left(\xi>\rho_{0}\right)$ by Lemma 3.2. Then, by the equation (3.17), we have

$$
\sigma\left(A_{r}(\xi)_{n-s}\right)= \begin{cases}\gamma+1 & n-s: \text { odd } \\ \gamma & n-s: \text { even, } r_{s}>0 \\ \gamma+2 & n-s: \text { even, } r_{s}<0\end{cases}
$$

for any $\xi>\rho_{0}$, which implies

$$
N_{f_{r}(\xi ; x)}=\sigma\left(A_{r}(\xi)\right)= \begin{cases}\gamma+1 & n-s: \text { odd } \\ \gamma & n-s: \text { even, } r_{s}>0 \\ \gamma+2 & n-s: \text { even, } r_{s}<0\end{cases}
$$

for any $\xi>\rho_{0}$ since $A_{r}(\xi)$ and $A_{r}(\xi)_{n-s}$ are equivalent over $\mathbb{R}$. Hence, by Lemma 3.5, we have

$$
\mathrm{N}_{\mathrm{f}_{r}(\xi ; x)}= \begin{cases}\gamma+1 & n-s: \text { odd } \\ \gamma & n-s: \text { even, } r_{s}>0 \\ \gamma+2 & n-s: \text { even, } r_{s}<0\end{cases}
$$

for any $\xi>\alpha_{r}$.

(ii) General case.

Let $\varepsilon_{0}$ be a positive real number and for any vector $v \in \mathbb{R}^{s+1}$, set

$$
\alpha_{v}^{\prime}=\max \left\{|\alpha| \mid \alpha \in \mathbb{C}, P_{v}(\alpha)=0\right\} .
$$

Clearly, we have $\alpha_{v}^{\prime} \geq \alpha_{v}$ for any $v \in \mathbb{R}^{s+1}$. Here, let us put $\rho_{0}^{\prime}=\alpha_{r}^{\prime}+\varepsilon_{0}$. Then, by Lemma 3.5. it is enough to prove the next claim.

Claim 1. For any real number $\xi>\rho_{0}^{\prime}$, we have

$$
\mathrm{N}_{\mathrm{f}_{r}(\xi ; x)}= \begin{cases}\gamma+1 & n-s: \text { odd } \\ \gamma & n-s: \text { even, } r_{s}>0 \\ \gamma+2 & n-s: \text { even }, r_{s}<0\end{cases}
$$

Proof. By the assumption that $\mathrm{g}_{\mathrm{r}}(\mathrm{x})$ is a separable polynomial of degree $\mathrm{s}$ and the fact that the non-real roots must occur in pair with its complex conjugate, there exists a real number $\delta_{0}$ such that for any vector $v=\left(v_{0}, \cdots, v_{s}\right) \in \mathbb{R}^{s+1}$ satisfying $|\mathrm{r}-v|_{0}=\max _{0 \leq k \leq s}\left\{\left|r_{k}-v_{k}\right|\right\}<\delta_{0}, g_{v}(x)$ is also a degree $s$ separable polynomial satisfying $N_{g_{v}}=N_{g_{r}}=\gamma$ by Proposition 3,

(S1) If a vector $v \in \mathbb{R}^{s+1}$ satisfies $|\mathrm{r}-v|_{0}<\delta_{0}$, then $g_{v}(x)$ is also a degree $s$ separable polynomial satisfying $\mathrm{N}_{g_{v}}=\mathrm{N}_{\mathrm{g}_{\mathrm{r}}}=\gamma$. 
Next, we put

$$
P(t)=\sum_{k \geq 0} x_{k}\left(t_{0}, \cdots, t_{s}\right) t^{k}=\operatorname{det} A(t)\left(A(t)=A\left(t_{0}, \cdots, t_{s}, t\right)\right)
$$

and let us consider $P(t)$ as a polynomial over $E_{1}=\mathbb{R}\left(t_{0}, \cdots, t_{s}\right)$ in $t$. Then, since $x_{k}\left(t_{0}, \cdots, t_{s}\right) \in$ $\mathbb{R}\left[t_{0}, \cdots, t_{s}\right]$ for any $k \geq 0$, there exists a real number $\delta_{1}>0$ such that for any vector $v \in \mathbb{R}^{s+1}$ satisfying $|r-v|_{0}<\delta_{1}$, we have $\left|\alpha_{r}^{\prime}-\alpha_{v}^{\prime}\right|<\varepsilon_{0}$ by Proposition 3.

(S2) If a vector $v \in \mathbb{R}^{s+1}$ satisfies $|r-v|_{0}<\delta_{1}$, we have $\left|\alpha_{r}^{\prime}-\alpha_{v}^{\prime}\right|<\varepsilon_{0}$.

Here, let $\xi$ be any real number such that $\xi>\rho_{0}^{\prime}=\alpha_{r}^{\prime}+\varepsilon_{0}$ and let

$$
\Omega\left(t_{0}, \cdots, t_{s}, \xi ; x\right)=\sum_{k=0}^{n} y_{k}\left(t_{0}, \cdots, t_{s}\right) x^{k} \in E_{1}[x]
$$

be the characteristic polynomial of the Bezoutian

$$
A\left(t_{0}, \cdots, t_{s}, \xi ; x\right)=M_{n}\left(f^{(n)}\left(t_{0}, \cdots, t_{s}, \xi ; x\right), f^{(n)}\left(t_{0}, \cdots, t_{s}, \xi ; x\right)^{\prime}\right) .
$$

Here, $f^{(n)}\left(t_{0}, \cdots, t_{s}, \xi ; x\right)^{\prime}$ is the derivative of

$$
f^{(n)}\left(t_{0}, \cdots, t_{s}, \xi ; x\right)=\sum_{k=0}^{n} z_{k}\left(t_{0}, \cdots, t_{s}\right) x^{k} \in E_{1}[x]
$$

with respect to $x$. Then, since $z_{k}\left(t_{0}, \cdots, t_{s}\right) \in \mathbb{R}\left[t_{0}, \cdots, t_{s}\right](0 \leq k \leq n)$, we also have $y_{k}\left(t_{0}, \cdots, t_{s}\right) \in$ $\mathbb{R}\left[t_{0}, \cdots, t_{s}\right](0 \leq k \leq n)$. Moreover, since $\xi>\rho_{0}^{\prime}>\alpha_{r}$, we have $\operatorname{det} A_{r}(\xi)=\operatorname{det} A\left(r_{0}, \cdots, r_{s}, \xi\right) \neq$ 0 .

By these arguments, we can also deduce that there exists a positive real number $\delta_{2}$ such that for any vector $v \in \mathbb{R}^{s+1}$ satisfying $|r-v|_{0}<\delta_{2}$, the characteristic polynomial $\Omega_{v}(\xi ; x)$ have the same number of positive and hence negative real roots with $\Omega_{\mathrm{r}}(\xi ; x)$ (counted with multiplicity), which implies $N_{f_{r}(\xi ; x)}=\sigma\left(A_{r}(\xi)\right)=\sigma\left(A_{v}(\xi)\right)=N_{f_{v}(\xi ; x)}$.

(S3) If a vector $v \in \mathbb{R}^{s+1}$ satisfies $|r-v|_{0}<\delta_{2}$, we have $N_{f_{r}(\xi ; x)}=N_{f_{v}(\xi ; x)}$.

Put $\delta=\min \left\{\delta_{0}, \delta_{1}, \delta_{2}\right\}>0$. Then, there exists a vector $\mathcal{w}=\left(\mathcal{w}_{0}, \cdots, \mathcal{w}_{\mathrm{s}}\right) \in \mathbb{R}^{s+1}$ such that

$$
\text { (a) }|\mathrm{r}-w|_{0}<\delta, \text { (b) } \mathrm{h}_{0}^{(w)} \mathrm{h}_{1}^{(w)} \cdots \mathrm{h}_{\mathrm{s}-1}^{(w)} \neq 0
$$

Here, we put $h_{s-k}^{(w)}=h_{s-k}\left(w_{0}, \cdots, w_{s}\right)$ for any $k(1 \leq k \leq s)$. In fact, since $h_{s-k}\left(t_{0}, \cdots, t_{s}\right)$ is a non-zero polynomial for any $k(1 \leq k \leq s)$ by Lemma 3.3 , the product $\prod_{k=1}^{s} h_{s-k}\left(t_{0}, \cdots, t_{s}\right)$ is also non-zero, which implies that there exists a vector $w \in \mathbb{R}^{s+1}$ satisfying (a) and (b).

Let $w \in \mathbb{R}^{s+1}$ be the vector as above. Then, since $|r-w|_{0}<\delta \leq \delta_{0}, g_{w}(x)$ is a degree $s$ separable polynomial satisfying $\mathrm{N}_{\mathrm{g}_{w}}=\gamma$ by (S1) and also, by (S2), we have $\alpha_{w} \leq \alpha_{w}^{\prime}<\alpha_{\mathrm{r}}^{\prime}+\varepsilon_{0}=$ $\rho_{0}^{\prime}<\xi$. Thus, by (b) and the case (i), we have

$$
\mathrm{N}_{\mathrm{f}_{w}(\xi ; x)}= \begin{cases}\gamma+1 & \mathrm{n}-\mathrm{s}: \text { odd } \\ \gamma & \mathrm{n}-\mathrm{s}: \text { even, } \mathrm{r}_{\mathrm{s}}>0 \\ \gamma+2 & \mathrm{n}-\mathrm{s}: \text { even, } \mathrm{r}_{\mathrm{s}}<0\end{cases}
$$


which, by (S3), implies

$$
N_{f_{r}(\xi ; x)}= \begin{cases}\gamma+1 & n-s: \text { odd } \\ \gamma & n-s: \text { even, } r_{s}>0 \\ \gamma+2 & n-s: \text { even, } r_{s}<0\end{cases}
$$

Since $\xi$ is any real number such that $\xi>\rho_{0}^{\prime}$, this completes the proof of Claim and hence the proof of Theorem 3.2 .

Proposition 5. Let $\mathrm{g}(\mathrm{x})=\sum_{i=0}^{s} \mathrm{a}_{i} \mathrm{x}^{\mathrm{i}}$ be a polynomial in $\mathbb{R}[\mathrm{x}]$ such that $\Delta_{\mathrm{g}} \neq 0$ and

$$
f(t, x)=x^{n}+t \cdot g(x)
$$

If $\mathrm{g}(\mathrm{x})$ is totally complex, $(\mathrm{n}-\mathrm{s})$ is even, and $\mathrm{a}_{\mathrm{s}}>0$ then $\mathrm{f}(\beta, \mathrm{x})$ is totally complex for all $\beta>\max \left\{\alpha \mid \Delta_{(f, x)}(\alpha)=0\right\}$.

Proof. We have to show that $f(\beta, x)$ has no real roots. Since $g(x)$ is totally complex we have that $\gamma=0 . N_{f(\beta, x)}=\gamma$ as $\beta>\max \left\{\alpha \mid \Delta_{(f, x)}(\alpha)=0\right\}$ and $a_{s}>0$, so $N_{f(\beta, x)}=\gamma=0$. Hence, $f(\beta, x)$ is totally complex.

Let $K:=\mathbb{Q}\left(t, a_{0}, \ldots, a_{s}\right)$ be the field of transcendental degree $s+1$ and $g(x)=\sum_{i=0}^{s} a_{i} x^{i}$. Then we have the following.

Corolary 2. Let $\mathrm{K}:=\mathbb{Q}\left(t, \mathrm{a}_{0}, \ldots, \mathrm{a}_{\mathrm{s}}\right)$ be the field of transcendental degree $\mathrm{s}+1, \mathrm{~g}(\mathrm{x})=\sum_{i=0}^{\mathrm{s}} \mathrm{a}_{i} x^{i}$ and

$$
f(t, x)=x^{n}+t \cdot g(x)
$$

For any value of $\left(\lambda_{0}, \ldots, \lambda_{s}\right) \in \mathbb{Z}^{s+1}$, if $\mathrm{g}\left(\lambda_{0}, \ldots, \lambda_{s}, x\right) \in \mathbb{Z}[\mathrm{x}]$ is irreducible and satisfies the conditions of the Eisenstein criteria, then $\mathrm{f}(\mathrm{x})$ is irreducible, over $\mathbb{Q}$.

We also note:

Remark 3.4. It can be verified computationally by Maple that if $\mathrm{n} \leq 9$ and $1 \leq \mathrm{s}<\mathrm{n}$ then the Galois group $\mathrm{Gal}_{\mathrm{K}}(\mathrm{f}, \mathrm{x})$ is isomorphic to $\mathrm{S}_{\mathrm{n}}$.

Remark 3.5. Polynomials in Eq. (3.18) for $\mathrm{s}=1$ and $\mathrm{t}=1$ has been treated by $Y$. Zarhin in [18] while studying Mori trinomials. It is shown there that the Galois group of $\mathrm{f}(\mathrm{x})$ over $\mathbb{Q}$ is isomorphic to $\mathrm{S}_{\mathrm{n}}$; see [18, Cor. 3.5] for details.

In general, if we let $K:=\mathbb{Q}\left(t, a_{0}, \ldots, a_{s}\right)$ be the field of transcendental degree $s+1$, for $1 \leq \mathrm{s}<\mathrm{n}$, then we expect that Gal $\mathrm{k}(\mathrm{f}) \cong \mathrm{S}_{\mathrm{n}}$ for all $\mathrm{n} \geq 1$. If true, this would generalize Zarhin's result to a more general class of polynomials.

\section{References}

[1] Oz Ben-Shimol, On Galois groups of prime degree polynomials with complex roots, Algebra Discrete Math. 2 (2009), 99-107. MR2589076 
[2] L. Beshaj, R. Hidalgo, S. Kruk, A. Malmendier, S. Quispe, and T. Shaska, Rational points in the moduli space of genus two, Higher genus curves in mathematical physics and arithmetic geometry, 2018, pp. 83-115. MR.3782461

[3] Lubjana Beshaj, Reduction theory of binary forms, Advances on superelliptic curves and their applications, 2015, pp. 84-116. MR3525574

[4] A. Bialostocki and T. Shaska, Galois groups of prime degree polynomials with nonreal roots, Computational aspects of algebraic curves, 2005, pp. 243-255. MR2182043

[5] Artur Elezi and Tony Shaska, Reduction of binary forms via the hyperbolic center of mass (2017), available at 1705.02618

[6] Paul A. Fuhrmann, A polynomial approach to linear algebra, Second, Universitext, Springer, New York, 2012. MR 2894784

[7] Ruben Hidalgo and Tony Shaska, On the field of moduli of superelliptic curves, Higher genus curves in mathematical physics and arithmetic geometry, 2018, pp. 47-62. MR.3782459

[8] David Joyner and Tony Shaska, Self-inversive polynomials, curves, and codes, Higher genus curves in mathematical physics and arithmetic geometry, 2018, pp. 189-208. MR 3782467

[9] A. Malmendier and T. Shaska, The Satake sextic in F-theory, J. Geom. Phys. 120 (2017), 290-305. MR3712162

[10] Andreas Malmendier and Tony Shaska, A universal genus-two curve from Siegel modular forms, SIGMA Symmetry Integrability Geom. Methods Appl. 13 (2017), Paper No. 089, 17. MR3731039

[11] Morris Marden, Geometry of polynomials, Second edition. Mathematical Surveys, No. 3, American Mathematical Society, Providence, R.I., 1966. MR.0225972

[12] Thomas Mattman and John McKay, Computation of Galois groups over function fields, Math. Comp. 66 (1997), no. 218, 823-831. MR1401943

[13] Shuichi Otake, Counting the number of distinct real roots of certain polynomials by Bezoutian and the Galois groups over the rational number field, Linear Multilinear Algebra 61 (2013), no. 4, 429-441. MR3005628

[14] _ A Bezoutian approach to orthogonal decompositions of trace forms or integral trace forms of some classical polynomials, Linear Algebra Appl. 471 (2015), 291-319. MR.3314338

[15] Shuichi Otake and Tony Shaska, Bezoutians and the discriminant of a certain quadrinomials, Algebraic curves and their applications, 2019, pp. 55-72.

[16] Q. I. Rahman and G. Schmeisser, Analytic theory of polynomials, London Mathematical Society Monographs. New Series, vol. 26, The Clarendon Press, Oxford University Press, Oxford, 2002. MR1954841

[17] Gene Ward Smith, Some polynomials over $\mathbf{Q}(\mathrm{t})$ and their Galois groups, Math. Comp. 69 (2000), no. 230, 775-796. MR.1659835

[18] Yuri G. Zarhin, Galois groups of Mori trinomials and hyperelliptic curves with big monodromy, Eur. J. Math. 2 (2016), no. 1, 360-381. MR3454107 